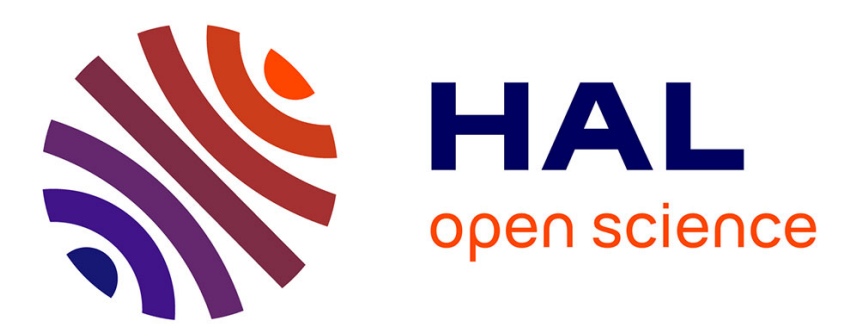

\title{
A generic and efficient emotion-driven approach toward personalized assessment and adaptation in serious games
}

Belkacem Mostefai, Amar Balla, Philippe Trigano

\section{To cite this version:}

Belkacem Mostefai, Amar Balla, Philippe Trigano. A generic and efficient emotion-driven approach toward personalized assessment and adaptation in serious games. Cognitive Systems Research, 2019, 56, pp.82-106. 10.1016/j.cogsys.2019.03.006 . hal-02071779

\section{HAL Id: hal-02071779 \\ https://hal.science/hal-02071779}

Submitted on 18 Mar 2019

HAL is a multi-disciplinary open access archive for the deposit and dissemination of scientific research documents, whether they are published or not. The documents may come from teaching and research institutions in France or abroad, or from public or private research centers.
L'archive ouverte pluridisciplinaire HAL, est destinée au dépôt et à la diffusion de documents scientifiques de niveau recherche, publiés ou non, émanant des établissements d'enseignement et de recherche français ou étrangers, des laboratoires publics ou privés. 


\section{Accepted Manuscript}

A generic and efficient emotion-driven approach toward personalized assessment and adaptation in serious games

Belkacem Mostefai, Amar Balla, Philippe Trigano

PII:

S1389-0417(18)30258-4

DOI: https://doi.org/10.1016/j.cogsys.2019.03.006

Reference:

COGSYS 833

To appear in:

Cognitive Systems Research

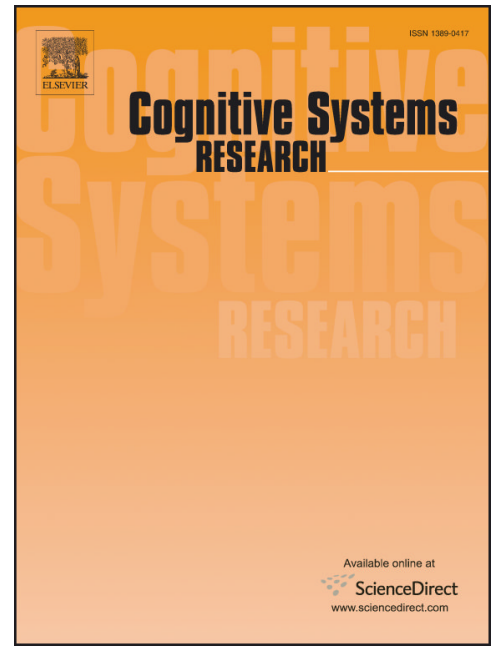

Received Date: $\quad 10$ June 2018

Revised Date: $\quad 15$ October 2018

Accepted Date: $\quad 8$ March 2019

Please cite this article as: Mostefai, B., Balla, A., Trigano, P., A generic and efficient emotion-driven approach toward personalized assessment and adaptation in serious games, Cognitive Systems Research (2019), doi: https:// doi.org/10.1016/j.cogsys.2019.03.006

This is a PDF file of an unedited manuscript that has been accepted for publication. As a service to our customers we are providing this early version of the manuscript. The manuscript will undergo copyediting, typesetting, and review of the resulting proof before it is published in its final form. Please note that during the production process errors may be discovered which could affect the content, and all legal disclaimers that apply to the journal pertain. 


\title{
A generic and efficient emotion-driven approach toward personalized assessment and adaptation in serious games
}

\author{
Belkacem Mostefai ${ }^{a^{*}}$, Amar Balla $^{\mathrm{a}}$, Philippe Trigano ${ }^{\mathrm{b}}$ \\ a Ecole national Supérieur d'Informatique ( E.S.I ex INI), LMCS \\ BP 68M Oued Smar, 16309, El Harrach, Alger, ALGERIE. \\ b Université de Technologie de Compiègne (U.T.C.), UMR CNRS 6599 Heudiasyc \\ BP 20529 60205, Compiègne, FRANCE
}

\begin{abstract}
Emotions have a major role in the player-game interaction. In serious games playing contexts, real-time assessment of the player's emotional state is crucially important to enable an emotion-driven adaptation during gameplay. In addition, a personalized assessment and adaptation based on the player's characteristics remains a challenge for serious games designers. This paper presents a generic and efficient emotion-driven approach for personalized assessment and adaptation in serious games, in which two main methods and their algorithms are proposed. The first one is a method for assessing, in real time, the player's emotion taking into account the personality type and the playing style of the player.The second one is an emotion-driven personalized adaptation method based on Markov modeling of dependency between the serious game events and the change in the player's emotional state. Therefore, the proposed approach has been evaluated by playing an affective vs. non-affective version of a serious game that we have developed to illustrate the applicability of the above-mentioned methods. The overall results showed that owing to our approach, a serious game become able to enhance its adaptivity toward playing outcomes and improve its overall playability.
\end{abstract}

Keywords: emotional state, affective serious game, assessment, adaptation, personality type, playing style.

\section{Introduction}

In the last decade, there has been a growing interest to study the affective aspects of serious games (Anolli, Mantovani, Confalonieri, Ascolese, \& Peveri, 2010; Aranha, Silva, Chaim, \& dos Santos Nunes, 2017; Connolly, Boyle, MacArthur, Hainey, \& Boyle, 2012; Hudlicka, 2008, 2009; Psaltis et al., 2016). The serious games concept is introduced by Abt (1987), who stated that "serious games have an explicit and carefully thought-out educational purpose and are not intended to be played primarily for amusement.". In the current research, it is well known that a serious game is a way to incorporate an educational scenario in a video game for conveying knowledge and skills. Moreover, there are games that do not have a direct educational focus, but can still be considered serious, such as games for rehabilitation. There are also games that can be fully educational even if it was designed with serious purpose, such as games for teaching geography or historical concepts. An adaptive serious game is a game that implements an adaptive gaming strategy (e.g., adaptive challenge, adaptive game mechanics, adaptive presentation) in order to adapt to a particular player's states,capabilities, needs, and interests (Hocine \& Gouaich, 2011; Ismailović, Haladjian, Köhler, Pagano, \& Brügge, 2012; Schrader, Brich, Frommel, Riemer, \& Rogers, 2017). Adaptive gaming methods are based on micro-adaptive approach (Kickmeier- Rust \& Albert, 2010), "which aim to auto-dynamically adapt game design elements to the needs of players and their changing characteristics just in time during gameplay" (Schrader et al., 2017). Thus, The player's characteristics have a high importance in adapting game learning processes and performance (Connolly, 2013; Frutos-Pascual \& Zapirain, 2017; Grappiolo, Cheong, Togelius, Khaled, \& Yannakakis, 2011; Rasim,

\footnotetext{
* Corresponding author, E-mail address: b_mostefai@esi.dz
} 
Langi, Munir, \& Rosmansyah, 2016). One important player's changing characteristic is the change in the player's emotional state which plays an important role in gaming experiences (Connolly et al., 2012; Kivikangas et al., 2011).

Current research on affective adaptation of games has mainly considered the change in the players' emotional state as the main player characteristic of adaptivity (Aranha et al., 2017; Bontchev, 2016a, 2016b; Schrader et al., 2017), this is due to the fact that "emotions are seen as a continuously progressing player characteristic that interacts with the game design and that changes during the time of gameplay"(Schrader et al., 2017). Therefore, an adaptation according to the change in the player's emotional state can improve the player's attitude to keep playing. It can also enhance the learning process (Brisson et al., 2012; Yannakakis, Isbister, Paiva, \& Karpouzis, 2014), and improve the use of the game over time (Anolli et al., 2010). Thus, an emotion-driven adaptive serious game, commonly called affective game, can adapt the gaming experience to the player's emotions. The primary aim of affective games is to improve the player's emotional engagement by recognizing and responding to player's emotional states during gameplay. In current affective game research, when talking about methods used to adapt to emotions, the subject of recognizing the player's emotional states is often closely associated. Therefore, assessing the player's emotional state during gameplay is one prerequisite to understand how and at what time there is a need to adapt (Schrader et al., 2017). Thus, as shown in Fig. 1, two main perspectives in affective game research can be distinguished : Emotion Recognition (ER) Emotion-driven Adaptation (EA).

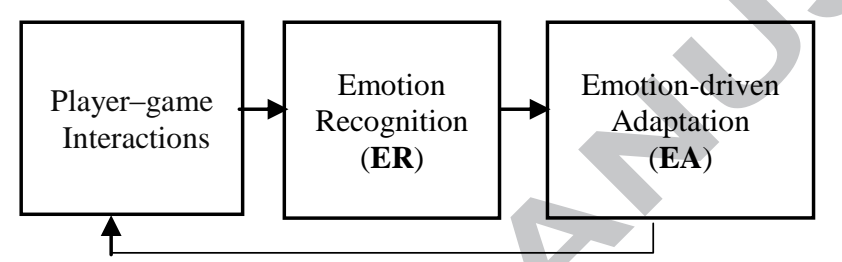

Fig. 1 Recognition and adaptation to player's emotions in affective game context : a symbolic representation.

Although there are several recent studies in the affective games field, little attention has been paid to propose a generic approach for both ER and EA taking into account the player's characteristics. In our opinion, we consider that player's emotions are in some way part of the interaction between the game events and the player actions. On that basis, we simply redefine a serious game as an inputs-outputs system, where inputs are the game events as the cause of emotions, and outputs are the player actions as the result of emotions. Hence, we propose a new approach that is able to recognize in real-time the player's current emotional state, and then adapt game events according to the change in the player's emotional state. In the following, we introduce first The ER method and then the EA method proposed for the emotion-driven approach.

Although the assessment of the player's emotions is important to design affective games, current approaches of ER still have certain limitations. Moreover, these approaches do not take account of the player's particular characteristics in recognizing emotions. After checking the literature, we concluded that the gameplay-based ER approach is less studied than others. The most important advantage of this approach is that it may lead to automatic and implicit measure of emotions without interrupting the gameplay or the need to use external tools besides the gaming devices. Thus, this approach has been considered a promising one to assess the players' emotional states, when playing a game (Schrader et al., 2017). In this paper, based on the gameplay-based ER approach, we propose a new method for recognizing during gameplay the player's emotional state. Morever, based on Frijda's emotion model (Frijda, 1987), we first define the player's emotional process, which includes two major steps: the appraisal of game events (evaluate how an event will affect the status of the current goal during gameplay), and the player's action tendencies (states of readiness to perform a given kind of action in order to establish, maintain, or disrupt the status of the current goal). Then, by inspiring from the studies of Power and Dalgleish (1997) and Perron (2005), we identify the mapping relation between different player actions and game goals status (e.g. goal achieved, goal blocked) on one side and different player's emotional states on the other side. This mapping relationcan be personalized according to two important individual player characteristics : the player's playing style (Hrabec, 2017; Mena, 2012), and the player's personality type(Menekse, Çagiltay, \& Ozcelik, 2015; Peever, Johnson, \& Gardner, 2012). Hence, this personalized mapping relation allow customasing the ER processes. To achieve this, different patterns of actions and game goals status are mapped to different emotional states for different pairs of playing style and personality type. In order to apply our proposed ER method in affective game, we conceive a personalized emotion assessment algorithm that can be easily implemented and embedded within in-game modules.

The most existing methods of EA adapt separately only one or two aspects of game features (Bontchev, 2016a; Schrader et al., 2017). Thus, we propose a new method to cover many game features in the adaptation. In this method, all game features that the change of their status can change the player's emotional state, are considered as game events. Thus, based on Frijda's emotion model (Frijda, 1987), in which it is claimed that 
emotions are caused by an appraisal of events, we model the mapping relation between game events and player's emotions through a Markov model (Fink, 2014). The obtained model defines for each event a transition probability matrix, which contains probabilities of transition from the current emotional state to another after the occurring of this event. Based on matrices transitions, we conceive an emotion-driven game adaptation algorithm that can intelligently manage game events in order to induce or to avoid a specific emotional state. As mentioned earlier, the playing style and personality type of the player are used to personalize the ER method. In the same way, we use these two characteristics to personalize Markov transition matrices of the emotion-driven game adaptation algorithm. Consequently, we conceive an emotion-driven personalized adaptation algorithm.

The proposed methods/algorithms for ER and EA seem to be a promising approach to identify what game features should be adapted for which emotion in which kind of player (player type and personality type). Finally, we can summarize our key contributions within this paper as follows:

(1) We propose a genric architecture of affective games, which includes an affective game module that provides a customized assessment and adaptation to the player's emotions.

(2) We propose a new generic method/algorithm for ER to assess, just in time during gameplay, the change in the player's emotional state.

(3) Based on the general probabilistic Markov model, we first model the change in the player's emotional state in accordance with game events occurrence. Then, we propose a new method and its algorithm for EA.

(4) We adopted two main player characteristics (personality type and playing style) to customize both ER and EA. Adopting personality and playing style is motivated by several studies, where it was claimed that emotional reactions are influenced by the playing style of the player (Arelíusarson; Holm, 2017; Lazzaro, 2008), and also by the personality type of the player (Menekse et al., 2015; Shiota, Keltner, \& John, 2006).

(5) We introduces a practical methodology to build the affective game module for any serious game.

(6) We designed and developed a new serious game, called DAPM (Drone against Programmed Machines), to better translate the actual player experience and then toor illustrate the applicability of the proposed methodology for developing the affective game module.

(7) We have performed an evaluation of the proposed approach within an experiment with real players (university students). The experiment was conducted by playing an affective (experimental group) vs. nonaffective (control group) version of DAPM. The obtained results have demonstrated the efficiency of our emotion-driven approach.

The organization of this paper is as follows. Section 2 presents a review of the existing approaches and methods for both emotion recognition and adaptation to emotion in the serious game playing contexts. Section 3 discusses related work. Afterwards, the proposed emotion-driven approach is outlined in section 4 which is subdivided into six subsections. In the first and the second subsection, we introduce a generic architecture for standard and affective games, respectively. In the third and fourth subsection, we explain the proposed method/algorithm for ER and EA, respectively. In the fifth subsection, we show how the personality type and playing style can be exploited to customize both ER and EA algorithms. In the sixth subsection, we define a practical methodology to build a game affective module for any serious game. Section 5 describes the performed experiments, their results and discussions. Finally, we end the paper with conclusions and future work perspectives.

\section{Emotion recognition and adaptation in the games playing contexts: a review of existing approaches.}

\subsection{Emotion Recognition (ER) approaches}

In ER research, a variety of methods to assess player's emotions during the time of gameplay were employed. These methods can be classified into three general categories: (1) ER using self-reported data, (2) ER based on physiological responses, and (3) gameplay-based ER. In the first category, methods based on self-reported data are used as an immediate way to identify the player's emotional states (Frommel et al., 2015; Yannakakis \& Togelius, 2011), where self-reports are administered to players between play sessions or during gameplay. In this last case, a questionnaire must be integrated into the game world (Frommel et al., 2015; Shute, 2011). There are many problems with using self-report questionnaires (Hoskin, 2012), but its major limitation is the interrupt of the gameplay and the individual understanding of emotion labels. Consequently, we can consider self-reported data as a noncontinuous measurement method that prevents the understanding of the relation between emotions and game events that happen during the time of gameplay. In contrast to the methods from the first ER category, the second category's methods enable a continuous measurement of the player's emotional state during gameplay. These methods exploit data about physiological responses to decide on emotions (Cacioppo, Berntson, Larsen, Poehlmann, \& Ito, 2000; LARSEN, BERNTSON, POEHLMANN, ITO, \& CACIOPPO, 2010). 
ER methods based on physiological responses can further be classified, according to the type of physiological data, into two main types: (1) methods based on internal physiological responses, and (2) method based on external physiological responses. In the first type, different player's emotional states are associated with different patterns of invisible physiological reactions in terms of electrodermal activity (Chanel, Rebetez, Bétrancourt, \& Pun, 2011; Drachen, Nacke, Yannakakis, \& Pedersen, 2010; Tognetti, Garbarino, Bonarini, \& Matteucci, 2010), cardiovascular signals (Dolar, 2014; Egan et al., 2016; Ravaja, Saari, Salminen, Laarni, \& Kallinen, 2006; Tijs, Brokken, \& IJsselsteijn, 2008), or electromyography data (Hazlett, 2006; Ravaja et al., 2006; Tijs et al., 2008). For instance, Chanel et al. (2011) revealed that an increase in heart rate when playing an ego-shooter game was associated with negative self-reported emotions, whereas a decrease was correlated with positive ones. Drachen et al. (2010) also observed a positive relation between high levels of electrodermal activity and frustration. Tijs et al. (2008) showed that an increased activity of the zygomatic muscle, which is indicative for smiling, when playing a Pacman game, corresponds to higher levels of self-reported positive emotions. In the second type, player's emotions are assessed based on visible physiological reactions in terms of facial expressions (Wiklund, Rudenmalm, Norberg, Westin, \& Mozelius, 2015), head movements (Shaker, Asteriadis, Yannakakis, \& Karpouzis, 2011), or Whole-body behavior (Bianchi-Berthouze, 2013; Brich et al., 2015; Kleinsmith \& Bianchi-Berthouze, 2013). For example, the FaceReader software in (Noldus., 2014) was adopted to compare facial expressions of emotion between players playing two different games (Wiklund et al., 2015).

The limitation of ER methods based on physiological responses, is that they are costly methods, which need the use of software and external data acquisition system. This software presents difficulties to the player when playing with. Moreover, Physiological measurements are repeatedly ambiguous (Schrader et al., 2017) as they have more than one meaning for interpreting player's emotions in game context.

To overcome the limitations of physiological response ER methods, some important works have proposed gameplay-based ER methods (Brich et al., 2015; Tijs et al., 2008). These methods evaluate the current state of player-game interaction to further understand and explain player's emotion. The player-game interaction state can be characterized by : the game events that occur (Pedersen, Togelius, \& Yannakakis, 2010; Ravaja et al., 2005; Ravaja, Turpeinen, Saari, Puttonen, \& Keltikangas-Järvinen, 2008), the most frequent game features that are used by players (Brich et al., 2015; Hastings, Guha, \& Stanley, 2009; McMahan, Parberry, \& Parsons, 2015), players' in-game behavior (Hastings et al., 2009; Yannakakis \& Togelius, 2011), or the conversational interaction between the player and the non-player character (Tijs et al., 2008). Controlling particular game events has been used to influence physiological responses that have been shown to have the capability to measure emotions (Ravaja et al., 2005; Ravaja et al., 2008). Pedersen et al. (2010) used artificial neural networks to learn the relation between player's gameplay characteristics as game features and the value of the player's emotional preference in a particular game. Furthermore, Yannakakis and Togelius (2011) suggested that player's in-game behavior during gameplay can be used as an important additional source of information to infer player's emotions. Two potential limitations can be considered in gameplay-based ER methods. The first limitation is the need to determine the relation between player-game interactions data and the player's emotional states, while the second concerns the requirements of huge amount of training data to model this relation. Thus, this paper introduces the emotion-driven approach to overcome the first limitation. The second limitation can be overcome by exploiting currently available technology to save and process massive amounts of information.

\subsection{Emotion-driven Adaptation (EA) approaches}

ER methods allow the recognition and the assessment of the player's emotions, which are used as inputs to the EA methods (see Fig. 1 in section 1). Generally, an EA method should be able to influence the user states through induction of desired states and mitigation of undesirable states, knowing that a scheme of desirable vs. undesirable states may be developed by the designer of the EA system (Fairclough \& Gilleade, 2012). In the context of an affective game, desired states are the positive emotional states, e.g. player contentment or interest, that have a positive influence on the player's gaming experience. However, undesirable states are negative emotional states, e.g. player frustration or boredom, that have a negative impact on the gaming experience.

Design methods for EA in affective game, are still a new area of research. Furthermore, in most recent studies, these design methods are the same ones used for adaptive video games. Recently, EA methods that have been used in both video and serious games are reviewed in (Bontchev, 2016a; Schrader et al., 2017). Based on these reviews, currently employed EA methods fall into two main approaches:

(1) adaptation of the gameplay mechanics.

(2) adaptation of representational aspects of the game.

EA methods of the first approach are organised into three groups: (1) adaptation of the gameplay difficulty level, (2) adaptation of the motivational gameplay rewards, and (3) adaptation of social gameplay elements. The first group, concerning the adjustment of gameplay difficulty which is used to constantly improve the player's emotional engagement (Liu, Agrawal, Sarkar, \& Chen, 2009). For instance, the gameplay scenarios' difficulties could be adapted by procedurally generating conflict scenarios adapted to the playing style and other indications 
of affective and cognitive states of the player (Yannakakis et al., 2010). Furthermore, a high level of gameplay which decrease the player's play challenge can lead to negative emotions (e.g, bored frustrated and depressed) (Schrader \& Nett, 2017; Wang \& Sun, 2011). The second group is related to the reward system in gameplay, which can affect the player's emotions, motivations and behaviors (Medler, 2011; Wang \& Sun, 2011). For example, playing with challenging difficulties may lead to boredom. Thus, a reward adaptive strategy can help the player to establish higher challenges or change the player's emotional state through rewards (Wang \& Sun, 2011). The third group, is refers to the social gameplay elements, e.g., chat and social media functionality, that allow social interaction between players (Kaye \& Bryce, 2012; Schrader et al., 2017). These elements can be adapted to enhance the emotional experiences of gaming, as for maintaining the interest emotion in playing, taking into account the player's preference, the game may be adapted by activating a functionality that enables the player to compete challenges with another player (Schrader et al., 2017).

In the second approach, EA methods focus on three main groups of the representation aspect of adaptation: (1) adaptation of audio-visual effects, (2) adaptation by alternating the gaming paradigms or layouts, and (3) adaptation of game's narrative progressions. The first group, provides an adaptation of the audio-visual effects through different methods: adjustment of the ambient light in a horror game (Grigore, Gavat, Cotescu, \& Grigore, 2008), adapting the game sound to game events (Gasselseder, 2014), and adapting visual representation, whether color or shape design of specific game objects like in-game message interfaces and the appearance of non-player characters (Schrader et al., 2017). The second group of adaptation strategy has been suggested by Schrader et al. (2017) who discussed the possibility of adapting a game using its world paradigm or its world layout. Within the game world paradigm the game-player interaction can be adapted by changing the gameworld exploration mode (open vs. linear mode). In the same way, open vs. linear game world layouts can be used to adapt different complexity levels of space layouts according a particular player's emotional state. The third group, concern the adaptive influence of length, complexity, and order of the learning content of the storyline and narrative aspects of a game. The representation of game's narrative progressions can be adapted to procedurally generate the narrative content (Yannakakis et al., 2010), to evoke emotional changes (Bopp, 2008) and to teach, in an efficient way, specific skills (Schrader et al., 2017).

Regarding the field of affective games, an EA method can be further designed using any one or combinations of the above mentioned adaptive methods, i.e., adaptation incorporates both mechanics and representational aspects of the game. However, the adaptive methods differ in their requirements and complexity, such as degree and time of adaptation (Schrader et al., 2017), in a way that not every EA is suitable for every kind of games. Furthermore, though the variety of EA methods, there is no detailed methodology to conceive and implement an algorithm for these methods. Little recent research proposes an adaptive method that can be adopted to develop an EA method for affective games. Recently, Schrader et al. (2017) highlighted that some existing adaptive game design generally provide only a low granularity in their degree of adaptivity, and it is still unclear what game features should be adapted for what emotion in what type of player. Further investigations into most approaches of both ER and EA methods are still ongoing (Bontchev, 2016; Karpouzis \& Yannakakis, 2016; Schrader, Brich, Frommel, Riemer, \& Rogers, 2017).

\section{Related work}

Many researchers have tried to deal with affective adaptivity of serious games by proposing different approaches, whether for ER or EA. Recently, Schrader et al. (2017) and Bontchev (2016a) present their own literature review of ER and EA methods applied in both video and serious games. The findings of these reviews emphasize that most of currently methods employed to recognize emotion have mainly adopted self-reported data ER approach or physiology-based ER approach, while a few of themes have considered the emotion-based gameplay approach for assessing the player's emotional states and embed emotional support within an affective game. The major limitation to recognize emotions using self-reported data is the interrupt of the gameplay, while the main disadvantage of physiology-based ER methods is that physiological measurements are frequently ambiguous. In addition, they are costly methods (which often needs additional sensor devices for recognizing emotion) often restricted to laboratory experiments. In contrast, the emotion-based gameplay approach seems to be a promising approach to embed the assessment of the player's emotional state (Schrader et al., 2017). With regard to EA methods, few publications are available in the literature reviews that apply recognized emotions for adapting in real time only some game features. Moreover, they did not provide a detailed methodology to explain how develop their EA methods. With regard to affective adaptivity of games, we find that the following studies are particularly interesting: (Karpouzis, Yannakakis, Shaker, \& Asteriadis, 2015), (Karpouzis et al., 2013) (Yannakakis \& Togelius, 2011) (Pedersen et al., 2010) and (Tijs et al., 2008).

Karpouzis et al. (2015) introduced the platform experience dataset (PED) that contains multiple modalities of data from players (in-game behavior data, game context representation data, player demographic data, selfreported experience data, and visual information data) of Infinite Mario Bros game, which is a variant of the Super Mario Bros game (Nintendo, 1985). PED is created to provide possibilities for player experience modeling 
approaches that can, in turn, yield affluent customization of games. However, it has some limitations. First, there is no predefined mapping or relation between gathered layer's data stored in the PED and the players' emotional states. Second, PED focuses on a particular game, while the gathered data cannot be generalized in all genres of games. Third, if we want to build a similar platform for a particular serious game, many tools need to be used in different ways to capture multiple modalities of player's data, which is a challenging task.

To educate young people on how to resolve conflicts within emotive experiences, an adaptive multiplayer game is designed in the context of the Siren project (Karpouzis et al., 2013). The aim of the Siren serious game is to teach productive conflict resolution strategies by producing an adaptive content. The adaptation process consists of procedurally generating the upcoming Minigame scenarios according to the player's immersion level (fully, high or low immersed). The immersion level is estimated by a visual analysis procedure of affective, cognitive and behavioral information from the player. The player's emotional and cognitive state are estimated based on the analysis of the player's facial expressions (recorded during the gameplay by a plain web camera mounted on top of the player's screen) combined with psychological observations and previously recorded player data. However, , the authors do not explain how the visual analysis procedure works. Also, there are no details about the nature of both psychological observations and previously recorded data and how they are combined with the facial features analysis for estimating the player's emotional states. Moreover, there are no experiments or procedures to validate, or evaluate the proposed approach. At last, their adaptation of the next mission has a limited effect on the playing outcomes because it was not done in real time, especially when the duration of the mission is quite long.

Yannakakis and Togelius (2011) proposed an approach, called experience-driven procedural content generation (EDPCG), to optimize player experience through optimizing game content. Player experience is considered as a function of cognitive and affective patterns and playing style of the player. To capture different types of data about the player's experience during gameplay, EPCG approach combines three different types of data: data expressed by players through questionnaires, data obtained from real-time recordings of physiological signals, and data obtained through the interaction between the player and the game. However, it is not explained how combine different data for capturing, in particular, the player's emotional state and, in general, the whole player experience.

Pedersen et al. (2010) proposes neural network based approach for predicting player's emotions. In this approach, the predictive model is trained using the following data: the parameters of controllable game features, gameplay metrics that are related to the actions performed by the player in the game, and six self-reported emotional states. This predictive model can be used to define player' preferences (desired emotions in combination with parameters of selected game features) from which the game optimizes next levels to meet those preferences. However, this predictive model has some limitations. First, the quality of the predictive model depends critically on quality of emotions used for training the model, where these emotions, which are primarily influenced by self-deception, are assessed using self-reported data related to the whole game session. Second, adapting next levels is valid only if the player's preferences remain fixed within a game session, otherwise, defining a real-time adaptation method is necessary. Third, this approach was not verified with studies on real players.

Tijs et al. (2008) investigated the relations between some player's emotional states (boredom, frustration and enjoyment) and one game feature (game speed). Where, self-reported emotional state emotional are obtained by pausing the gameplay and asking. In parallel, a series of emotional physiological data were recorded during gameplay to distinguish between the states of enjoyment, frustration and boredom. Then, they manipulated the game's speed to influence the player's emotional state. The proposed mapping between only one game feature and emotions is very straightforward and does not work properly in the case where a combination of various main features are necessary for winning one game. Furthermore, this approach requires physiological devices for recognizing emotions.

In general, there is at present large agreement that affective gaming is not a new topic; however it is still relatively undeveloped. Thus, there is no generic approach for providing personalized ER and EA in the games playing contexts. Furthermore, player traits are often not taken into account in the existing approach. Concluding their reviews of the research literature, Schrader et al. (2017) and Bontchev (2016a) suggested that future research should investigate various ways for improving the gaming experience through recognition of the player's emotional state and applying it for dynamic adaptation of game features. Schrader et al. (2017) have stressed the importance of gameplay for recognizing emotion, despite the fact that it needs assumptions about the mapping of in-game data and emotions. What is more, Bontchev (2016a) have highlighted that "dynamic models of player behaviour focused on tracking and monitoring of player interactions can be used for both recognition of player's emotions and inducing appropriate emotions during gameplay". Based on these findings, this paper proposes a generic and efficient emotion-driven approach, in which, the mapping relation, between the player actions and different games goals status one one side, and the the player's emotional states on other side, are modeled based on Fridja's emotion model (Frijda, 1987) and by inspiring from Power and Dalgleish (1997) and 
Perron (2005) studies. While, the change in the player's emotional state is modeled, using a Markov probabilistic model. The proposed approach is experimentally validated by playing two versions of a serious game called DAPM, including and excluding emotional adaptivity (affective vs. non-affective game). DAPM is a game that we have developed for training players on the evaluation of computer program written in Pascal programming language. The analysis of collected data indicates that players do seemingly show a higher engagement by having an emotion-driven personalized adaptation.

\section{A generic emotion-driven approach for assessment and adaptation in serious games}

The main aim of the emotion-driven approach is to enhance the player's emotional engagement, during gameplay, by increasing his/her positive emotions and reducing the negative ones. Positive emotions are desirable emotional states that encourage play and have postive impact on the gaming and learing outcomes.Hence, they should be maximized in order to keep the player engaged. Negative emotions are undesirable emotional states that discourage play and have negative impact on the gaming and learing outcomes.Hence, they should be minimized for not abandoning the play. In a nutshell,we want to determine for any serious game:

- A generic ER method for assessing the player's emotional state just in time during gameplay. where, emotions should be recognized without the need to interrupt the play or using external tools besides the gaming devices.

- A generic EA method for adapting game's features according the change in the player's emotional state. where all game features that can influence the player's emotional state should be considered in the adaptation.

In order to identify the criteria needed for these methods, we will first identify an adequate definition and a generic architecture of serious games. Then, we will show the proposed methods and their algorithms for assessing the player's emotions during gameplay and adapting the game according to the change in the player's emotional state.

\subsection{A generic architecture of serious games}

We search a definition that allowing the identification of a generic architecture of serious games. Among existing definitions of games that can be adopted to define serious game, we have chosen this definition given by Szilas and Acosta (2011): "A game is a dynamical system of signs in which the player acts, independently of any consequence outside the system, in order to reach a goal assigned by the game.". Therefore, "a sign is considered a link between a physical configuration of the game world and its interpretation (or representation) in the mind of the player".

This definition was initially given for educational games. However, it is very appropriate for defining serious games from a generic aspect because it is a generic description that include the notion of dynamical system. A game dynamical system contains two components (see Fig. 2) :

- The game mechanics : describe how the game events evolves (outputs), according to the player's action. It is composed of a specific component designed for the game, and of an environmental component.

- The playing rules: define what the players are allowed to do in the game (inputs). So, the playing rules act as a filter between all possible actions of the player and the allowed actions provided to the game mechanics.

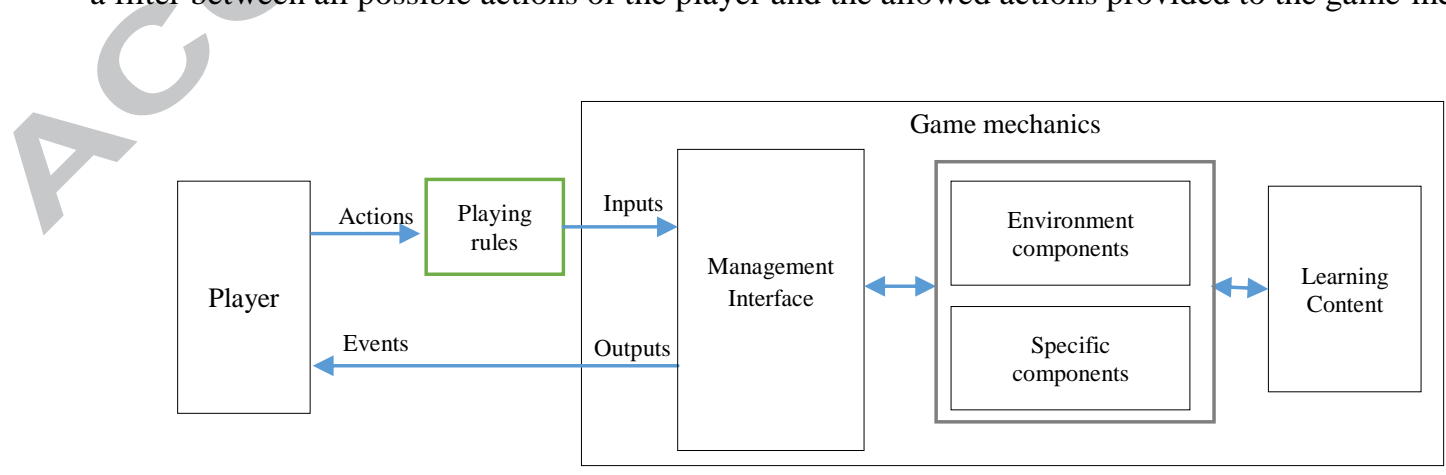

Fig. 2 A generic architecture of serious games, inspired and adapted from (Szilas \& Acosta, 2011)

In this paper, we are interested by the abovementioned game definition for the following two reasons. First, the author simplifies the definition of game as inputs and outputs in a dynamical system. Inputs are all possible player actions, while outputs mean the game events provided by the game mechanics. Therefore, The author showed a simple and direct relation between inputs and outputs. Secondly, in the light of this definition, the 
author formally distinguished between four strategies for produce serious learning in-game. Thus, we can exploit one or more of these strategies for improving the educational purposes in the game context.

In our view, we can adopt the same definition of educational games to define serious games. Thus, we can define a serious game as "a dynamical system of signs in which the player acts, independently of any consequence outside the system, in order to reach a fun and serious goals assigned by the game". Where, the dynamic system is composed of the game events (generated by the game mechanics) and the player actions (controlled by the playing rules). Also, we consider a sign as "a link between a physical configuration of game features and its interpretation, mental and emotional, in the mind of the player".

To recognize player's emotions during gamplay, most of previous research have employed either selfreported data method, which interrupt the gameplay, or physiological method, which require additional components (to measure physiological response) that disturb the player or impede the playing progress. This stresses the need for analysing the player-game interaction to assess in real-time the player's emotional state without the need to interrupt the gameplay or disturbing the rhythm of the play.

Based on the above findings ( the generic definition and architecture of serious games), we will introduce, in the next section, our proposition for a generic architecture of affective games.

\subsection{An generic architecture of affective games}

Fig. 3 describes our proposed architecture of affective games, which adapts its features in real time during the gameplay according the change in the player's emotional state. This architecture consists of three main modules: The game affective module, the game mechanic module and the playing-rules module.

The game affective module includes two submodules: the personalized emotion assessment module and the emotion-driven personalized adaptation module. The personalized aspect means that the player's emotional states are assessed according to the personality type and the playing style of the player.

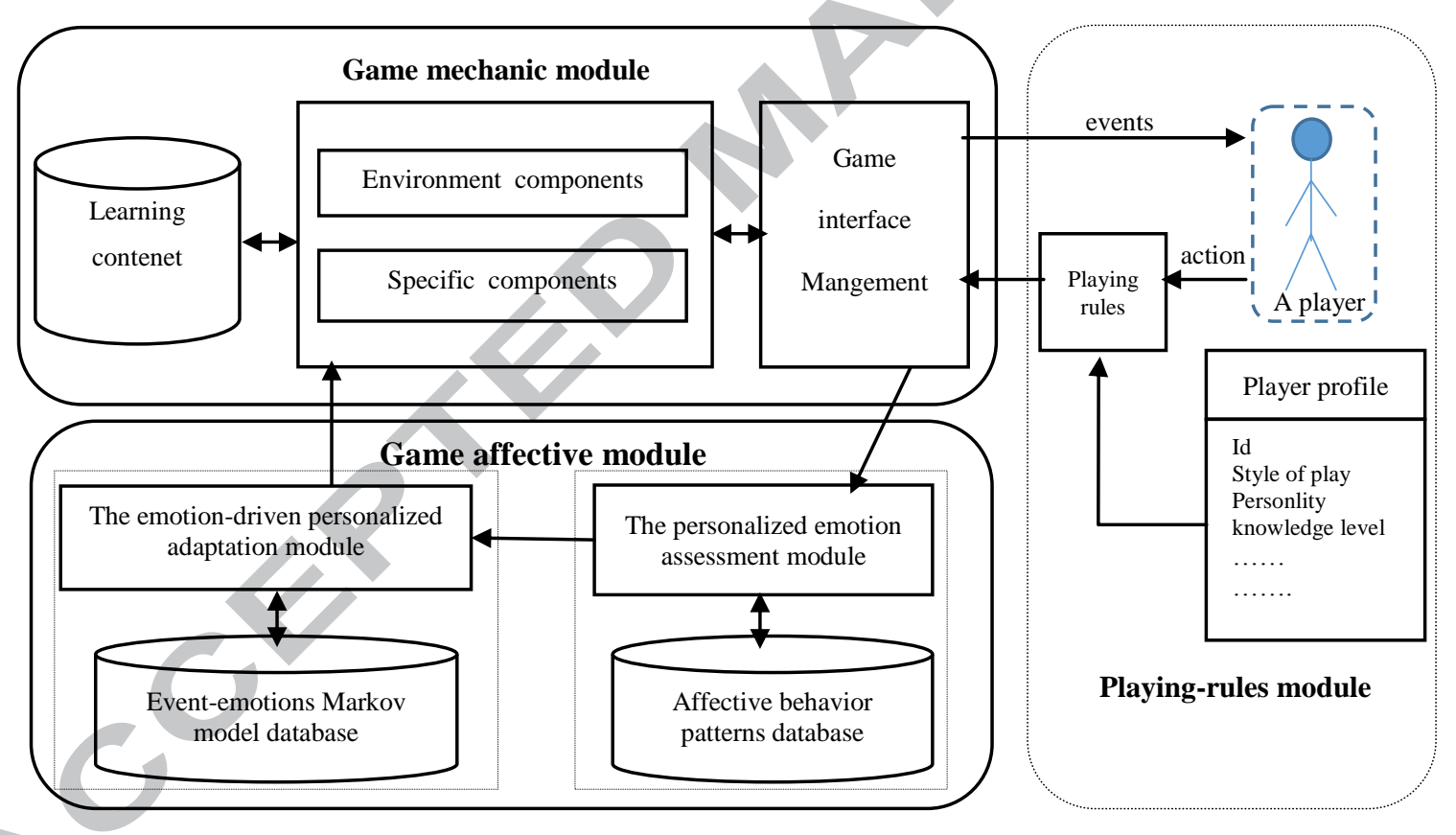

Fig. 3 An architecture for affective games

When the player performs an action, the playing rules module controls if this action is allowed, if so, then the game mechanics module runs this action. Right after that, the game adaptation module invokes the personalized emotion assessment submodule that exploits the affective behavior patterns database to estimate the actual player's emotional state, then it invokes the emotion-driven personalized adaptation submodule. If an adaptation is necessary, this last module exploits the event-emotions Markov model database to smartly manage the upcoming game events in order to evoke a positive emotion or to avoid a negative one. Hence, the game mechanics will be requested to execute the concerned events.

The affective behavior patterns database contains patterns of (personality type, playing style, an emotional state, a goal-status, and an action tendency) gathered from player-game interaction training data. The event-emotions Markov model database stores transition probability values of the change in the player's emotional sates according to each game event. The full details about each submodule will be explained later. In the next section, we will show our proposition to assess the change in the player's emotional state during gameplay. 


\subsection{The proposed ER method/algorithm}

As mentioned earlier, we considered serious game as inputs (player actions) / outputs (game events) of a dynamic system. From this point of view, we want to formally describe the relation between the game events (cause of emotion) and the player actions (consequence of emotion). The Frijda's model of emotions (Frijda, 1986, 1987, 2007), serves this purpose. In this model, the relation between an individual (the player in our context) and the environment (the game world in our context) is at centre stage to define emotion as highlighted in (Mesquita, 2016). Thus, emotion is considered as a cognitive process in which the appraisal of an event can trigger an emotion that ready the player for action. Also, the emotional process includes, inter alia, two major steps : the appraisal of events, the tendency to action.

\subsubsection{Emotion and action tendency}

The appraisal of an event means a cognitive evaluation to estimate how the event will affect the status of environment, so, this cognitive evaluation (event affect) elicits an emotional state which causes a tendency to action. The action tendency is state of readiness to perform a given kind of action in order to establish, maintain, or disrupt a relation with the status of the environment. Frijda (1987) showed that many emotions can be defined by different modes of action tendency, for example, anger is the urge to attack or, more properly, the urge to regain freedom of action and control, fear is the urge to separate oneself from aversive events. As shown in Table 1, Perron (2005) proposed to characterize some prototypical player's emotional states based on the principle of action tendency.

Table 1 Example of the link between some emotional states and their action tendencies, adapted from (Perron, 2005).

\begin{tabular}{|l|l|}
\hline Emotional state & Typical action tendencies \\
\hline Interest & a tendency to attend, to pay attention or to observe well and understand the situation. \\
\hline Enjoyment & a tendency to interact or to engage in consummatory activities. \\
\hline Worry & a tendency to turn toward an object. \\
\hline Fear & a tendency to move or to run away. \\
\hline Surprised & a tendency to interrupt an action or to freeze in order to reorient myself. \\
\hline Anger & a tendency to move against. \\
\hline Frustration. & a tendency to move in an antagonistic way or to redo the same actions all over again \\
\hline
\end{tabular}

In the games playing contexts, the environment of a player is the game-world. On the one hand, any game proposes goals to a player according to a specific situation (Lankoski, 2012). On the other hand, it has been shown that emotions depend on the player's appraisal in a given game situation (Perron, 2005). Thus, we can characterize a game situation by the status of the current given goal, which is provided by the game mechanics. Based on this finding, with consideration of the Frijda's model of emotions, we suggest that during the course of an event, the player's emotional state will arise from his/her evaluation of the event's effect on the status of the current goal. This current emotional state gives a player the tendency to prefer certain actions over others in order to reach the current goal, to progress towards a main goal or to maintain the status of the current goal. Briefly, the status of the current goal, guides the player to select an action from a set of action-possibilities related to the action tendency induced by an emotional state.

In the next subsections, we will show how the status of a goal can affect the choice of an action among others induced by the same emotional state.

\subsubsection{Link between emotion, goals status and action tendencies}

Several research studies show that emotion is the main factor that gives a player the tendency to perform a particular action over others (Damasio \& Sutherland, 1994; Perron, 2005; Steunebrink, Dastani, \& Meyer, 2009). According to (Steunebrink et al., 2009), games are typically about decision-making (for choice actions) which is guided by both emotions and goals. Thus, the choice of an action that is more useful in a given situation among others depends on the current goal status. The current goal status influences, in the first time, the cognitive appraisal of an occurring event, and then it influences the tendency to perform a particular action over others. In (Power \& Dalgleish, 1997), a model of emotion has been suggested, where different basic emotions are linked to different goals status and to their action tendencies. Table 2 shows an example of the relation between three elements: the emotional states, the goal status and the typical action tendencies. Where it can be noticed that the same emotional states do not necessarily match the same typical action tendencies. The reason for this is that there is no consensual definition of action tendency in the literature (Steunebrink et al. 2009). An action tendency depends on the value of the current emotional state and the status of the current goal. In the game 
playing context, we suggest that if the player assesses an event as constituting a barrier to current goal, then he /she feels a negative emotional state. If the player assesses an event as relevant to current goal, then he feels a positive emotional state. The current goal status is the main factor that pushes the player to choose specific action tendency among others. Thus, we argue that typical action tendencies of a game can be identified with the help of a game expert (e.g. game designers and educational experts) and validated by an experiment with real players.

Table 2 Example of relation between emotional state, goal status and action tendency, adapted from (Power \& Dalgleish, 1997)1

\begin{tabular}{|c|c|c|}
\hline Emotional state & Goal status & Typical action tendency \\
\hline $\begin{array}{c}\text { Happiness/ } \\
\text { Enjoyment }\end{array}$ & goal achieved & a tendency to avoid risk \\
\cline { 2 - 3 } & progress towards a goal & a tendency to continue with the goal. \\
\hline \multirow{2}{*}{ Fear } & goal is in great danger & a tendency to do avoidance behavior \\
\cline { 2 - 3 } & goal is in danger & $\begin{array}{c}\text { a tendency to freeze in place } \\
\text { specificity of game purpose }\end{array}$ \\
\hline Sadness / Frustration & goal losed or goal failure & a tendency to revenge \\
\hline Anger & a goal is blocked or frustrated & a tendency to do avoidance behavior \\
\hline Disgust & goal status is far from the goal ending status &
\end{tabular}

\subsubsection{An emotion assessment algorithm}

The aim is to assess the player's emotional state just in time during gameplay. Based on the precedent findings from the two last subsections, we propose to present the linking between emotion (the player's emotional state), cause of emotion (a game event), and consequences of emotion (the player's action) as shown in Fig. 4. When an event occurs, the player evaluates how this event affects the current goal status. This cognitive evaluation raises an emotional state of the player that causes a tendency to action. Thus, the player performs the appropriate action to carry out event or progress to the current game goal, after which new event

will be done.

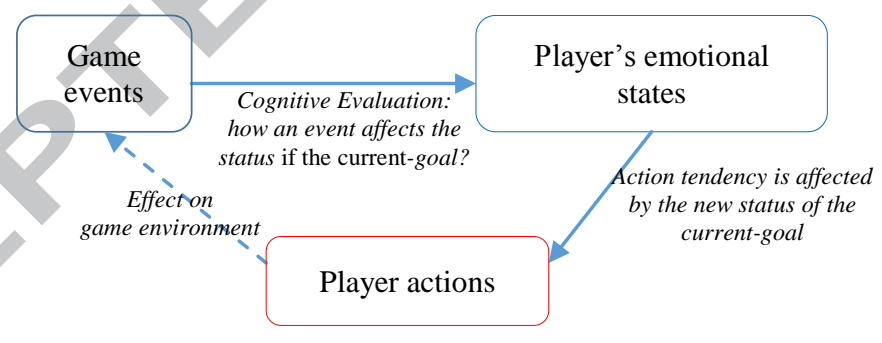

Fig. 4 Relation between the game events, the player's emotional states and the player actions in game context.

The analysis of the action patterns performed by the player after the occurring of an event taking into account the previous status of the current goal and the previous player's emotional state will enable to map different player's emotional states and goals status into specific player's action tendencies. It is important to note, if the serious game does not have clear goals, the help of the game designer expert is required to identify an implicit and explicit game goals and sub-goals to achieve the gaming missions. Fig. 5 illustrates a mapping relation between the player's emotional state, the status of the current goal, and the player's action. Thus, an emotional state can be assessed, in real time during gameplay, by investigating this mapping relation as shown in

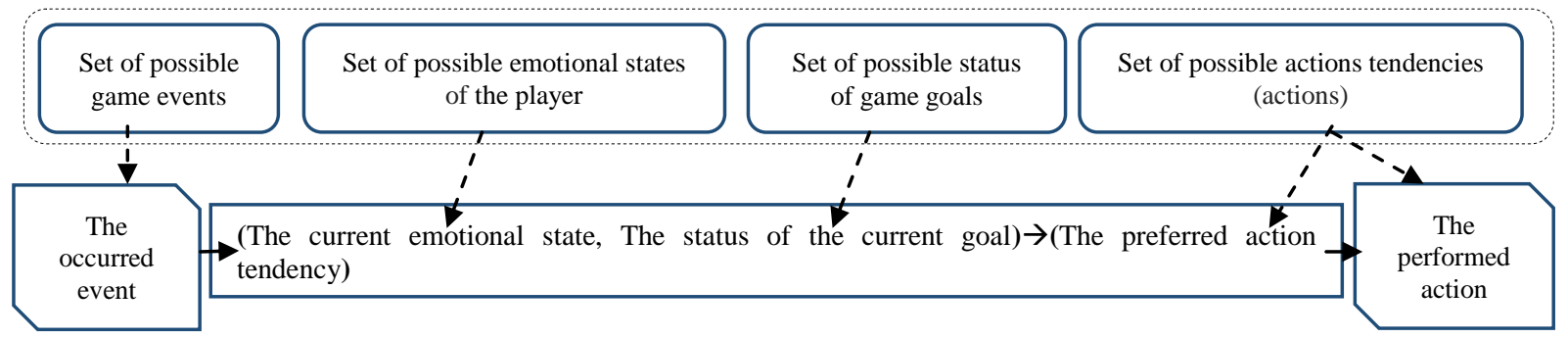


Algorithm 1.

Fig. 5 The mapping relation between emotional states/goal status and action tendencies

Games can elicit a wide variety of emotions. Since there are different kinds of games, there exist different emotional states according to the specificity of the serious game genre. Thus, in order to propose an algorithm to estimate in real time during gameplay the player's emotional state, we propose the definition of data structures and functions related to this specific game as follows:

- Defining a set of all events : $\quad$ Events $=\left(v_{1}, \ldots, v_{i}, \ldots . v_{n}\right)$

- Defining a set of all actions: Actions $=\left(a_{1}, \ldots, a_{i}, \ldots . a_{m}\right)$

- Defining a set of all possible emotional states: Emotions $=\left(e_{1}, \ldots, e_{i}, \ldots . e_{m}\right)$

- Defining a set of all game goals, including possible subgolas: $\quad$ goals $=\left(g_{1}, \ldots, g_{i}, \ldots . g_{p}\right)$

- Defining a set of all possible status of goals: $\quad$ goalstatus $=\left(g s_{2}, \ldots, g s_{i}, \ldots g s_{n}\right)$

- Defining "Fat" a mapping function that gives all actions tendencies for each emotional state :

Fat $\left(e_{i}\right) \rightarrow\left(a t_{1}, \ldots, a t_{i}, \ldots a t_{m}\right), e_{i} \in$ Emotions,$a t_{i} \in$ Actions.

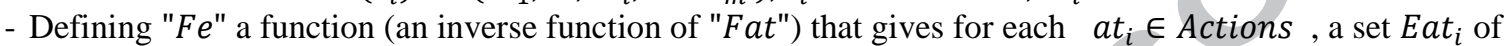
emotions that can urge this action. $F e\left(a t_{i}\right) \rightarrow E a t_{i}=\left(e m_{1}, \ldots, e m_{i}, \ldots . e_{m n}\right)$,

em $_{i} \in$ Emotions, at $t_{i} \in$ Actions .

- Defining "Fegs" a mapping function that determine the corresponding action tendencies for each emotional state and for each goal status: $\quad F e g s\left(e m_{i}, g_{i}, g s_{i}\right) \rightarrow\left(a t_{i}\right)$

Thus, we conceive the following algorithm to assess in real time during gameplay the player's emotional state.

Algorithm 1 An emotion assessment algorithm

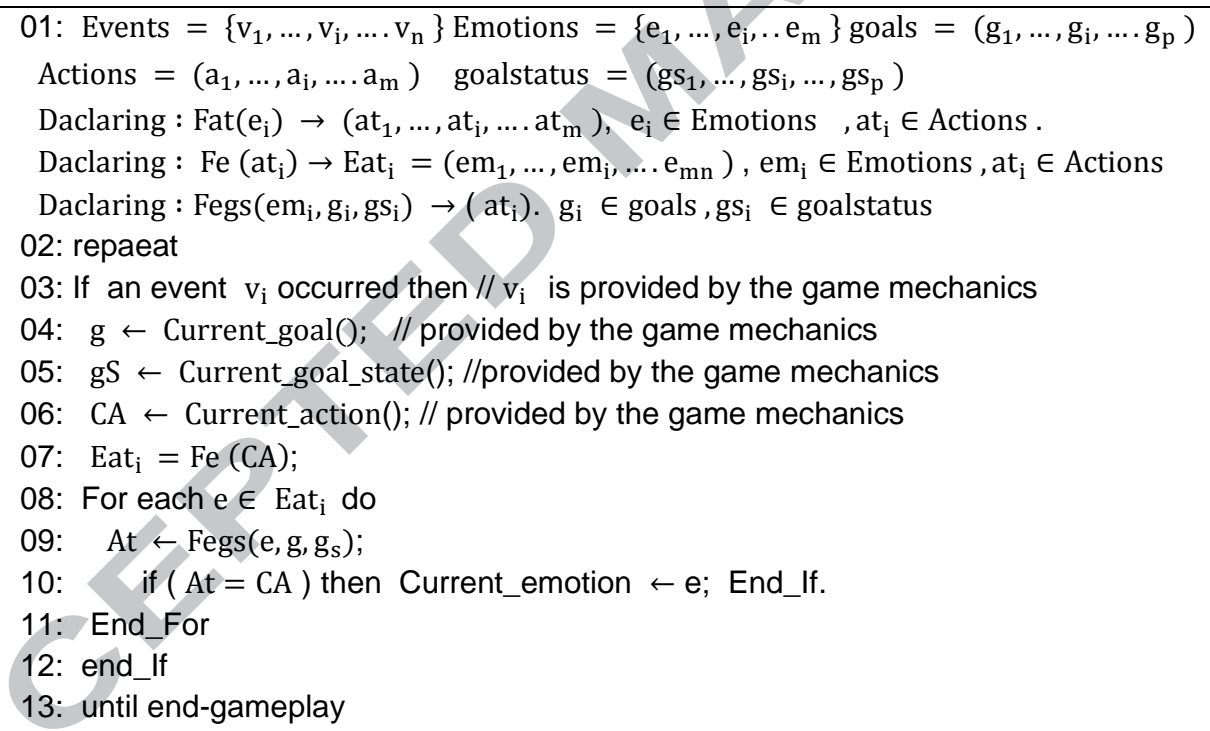

\subsection{The proposed EA method/algorithm}

To assess the player's emotional state, in the context of a specific game, we proposed an emotion assessment algorithm. Player's emotional state can unconsciously change from one state to another according to one or more events. Identifying how events cause the change in the player's emotional state has a clear practical importance to adapt the game according to the player's emotion. To this end, the following steps are performed:

- First, determining the relation between the change of the player's emotional states and the game events occurred during gameplay.

- Then, modeling the changes in the player's emotional state in accordance with game events' occurrence.

- Finally, conceiving an algorithm that enables adaptation according to the change in the player's emotional state.

\subsubsection{Relation between the change in the player's emotional state and the in-game events occurrences}

In order to model the relation between game events and the change in the player's emotional state, in the context of a specific game, we suggest on analyzing the dynamic change in the player's emotional state according to the occurence of game events. That is, for each event, we determine the player's emotional state

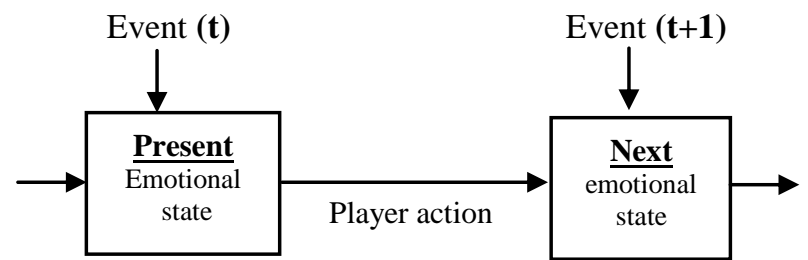


before and after the end of the action performed by the player to carry out this event. At any time, during the gameplay, it is possible to identify which event occurred last, because the game mechanic generates it. Therefore, the current player's emotional state can be assessed thanks to our emotion assessment algorithm which was introduced earlier.

Fig. 6 Symbolic representation of the change in the player's emotional state after the occurring of an event.

Fig. 6 describes the change of player's emotional state, at a specific step in time during gameplay. We observe the current emotional state of a player, at this time, the game mechanics execute an event, and then the player chooses an action to carry out this event. Therefore, the present player's emotional state evolves to a possible next player's emotional state at a subsequent step in time. Noting that both present and next player's emotional state may have the same value. That is, the player's emotional state stays the same. From the point of view of Markov chain theory, the transition of player's emotion from one state to another depends only on the occurring event.

\subsubsection{A Markov modeling of the change in the player's emotional state}

On one hand, we suggest that there are a set of finite events for any game, and a set of finite player's emotional states which can elicited by the game for any player. Furthermore, the player's emotional states can change from one state to another according one or more events. On the other hand, Markov chains offer ideal conditions for modelling a certain kind of situations (emotional state of the player in the game playing context) depending on random variables ( game events). Therefore, we can use Markov model in a standard way to track the changes in player's emotional state arising due to each game event. The change in a Markov model state is called transition.

In our study, it is assumed that any game has a known number of events $\left(v_{1}, \ldots v_{k}, \ldots v_{m}\right)$ and $\boldsymbol{N}$ player's emotional states $\left(e_{1}, \ldots e_{i}, . . e_{n}\right)$. We construct, for each event, a Markov chain of $\boldsymbol{N}$ states. The transition graph for a Markov chain, is a directed graph $\mathrm{G}$ which has a set of nodes and a set of arcs. Each node corresponds to a state of the Markov chain, and $\mathrm{G}$ contains $\operatorname{arc}\left(e_{i}, e_{j}\right)$ if the probability, that a player's emotional state changes from emotion $e_{i}$ to emotion $e_{j}$, is strictly positive.

Associated with a Markov chain, a transition matrix with $N$ emotional states is an $N \times N$ matrix where the entry in row $i$ and column $j$ is the probability that a player's emotional state will be changed from emotion $e_{i}$ into emotion $e_{j}$ after the occurrence of an event $v_{k}$. Thus, to model the change in the player's emotional state in accordance with game events based on Markov model, we consider a set with finite number of game events, where each event corresponds to a finite Markov Chain with $N$ emotional states, $N \geq 2$. Thus, we have the following denotations:

- $P_{i j}$ the transition probability from state $e_{i}$ to state $e_{j}$, where $i, j \in(1,2, \ldots, n)$; $-E_{v_{k}}=\left(\begin{array}{ccc}P_{11} & \cdots & P_{1 n} \\ \vdots & \ddots & \vdots \\ P_{n 1} & \cdots & P_{n n}\end{array}\right)$ The transition matrix of the Markov Chain related to the event $v_{k}$.

Since the transition from a state to some any other state (including itself) is a certain event, we have the basic properties of the transition probability matrix of a Markov chain that is defined as follows:

$$
0 \leq P_{i j} \leq 1, \text { and } \sum_{j=1}^{j=n} P_{i 1}=1 ; \forall i, j \in(1, \ldots, n)
$$

P $\left(e_{i} \mid e_{j}, v_{k}\right)$ the $P_{i j}$ when the event $v_{k}$ occurred.

In practice, to calculate transition probabilities matrix of Markov Chain for all game events, we need first to obtained enough count data (gathered during several gameplay sessions) about the sequence of the occurrence of emotional states for each game event. Based on these count data, we compute for each event the number $N_{i j}$ that denote the number of transitions from an emotional state $e_{i}$ to an emotional state $e_{j}$. Thus, we can estimate the transition probability from state $e_{i}$ to state $e_{j}$, denoted $P_{i j}$, using the following formula:

$$
P_{i j}=\frac{N_{i j}}{\sum_{j} N_{i j}}
$$

Thus, the probability of transition $P_{i j}$ is equal to the proportion of transitions started in emotional state $e_{i}$ and ended in emotional state $e_{j}$ as a proportion of all transitions in that started from the emotional state $e_{i}$.

Let us now see how the above formula works for a simple example. We consider the player's emotional state changes between two states $E=\left\{e_{1}, e_{2}\right\}$ according to the event $v_{1}$. Let $N_{e_{i} e_{j}}$ be the number of transitions from 
$e_{\mathrm{i}}$ to $e_{\mathrm{j}}, i, j \in(1,2)$, which come up from count data gathered during several gameplay sessions. With the following values of $N_{e_{i} e_{j}}$ related to the event $v_{1}: N_{e_{1} e_{1}}=30 ; N_{e_{1} e_{2}}=70 ; N_{e_{2} e_{1}}=40 ; N_{e_{2} e_{2}}=60 ; \quad$ We would compute transition probabilities matrix of Markov Chain related to this event.

So let us calculate $P_{i j}$ using this above mentioned formula:

$$
\begin{aligned}
& P_{11}=\frac{N_{e_{1} e_{1}}}{\sum_{j} N_{e_{1} e_{j}}}=\frac{30}{30+70}=0.3 ; P_{12}=\frac{N_{e_{1} e_{2}}}{\sum_{j} N_{e_{1} e_{j}}}=\frac{70}{30+70}=0.7 ; \\
& P_{21}=\frac{N_{e_{2} e_{1}}}{\sum_{j} N_{e_{2} e_{\mathrm{j}}}}=\frac{40}{40+60}=0.4 ; P_{22}=\frac{N_{e_{2} e_{2}}}{\sum_{j} N_{e_{2} e_{j}}}=\frac{60}{40+60}=0.6 ;
\end{aligned}
$$

Thus, the transition graph associated with Markov chain related to $v_{1}$ is represented as shown below:

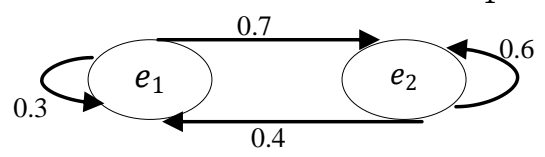

We assume that at each time the player's emotional state is either state $e_{1}$ to $e_{2}$. So, if the current emotional state is $e_{1}$, and if the event $v_{1}$ occurred, then Markov chain will provide us with the transition probability of the next player's emotional state which is the same state with probability 0.3 or the state $e_{2}$ with probability 0.7 . In other cases, if the current emotional state is $e_{2}$ and if the event $v_{1}$ occurred, then Markov chain informs us with probability 0.6 that the next state is $e_{1}$ or still in the same state with probability 0.4 .

The previous transition graph for Markov chain will be transformed into a transition matrix.

$$
E_{-v_{1}}=\left[\begin{array}{ll}
0.3 & 0.7 \\
0.4 & 0.6
\end{array}\right]
$$

The transition probabilities associated with various state changes can write as follows:

$$
\mathrm{P}\left(e_{1} \mid e_{1}, v_{1}\right)=0.3 ; \mathrm{P}\left(e_{1} \mid e_{2}, v_{1}\right)=0.7 ; \quad \mathrm{P}\left(e_{2} \mid e_{1}, v_{1}\right)=0.4 ; \mathrm{P}\left(e_{2} \mid e_{2}, v_{1}\right)=0.6
$$

Generally, the transition matrices can be calculated through empirical test that allow the tracking of the change in the player's emotional state arising due to different game events.

\subsubsection{An emotion-driven adaptation algorithm}

It was explained earlier that the player's actions and game goals status is relevant for assessing the player's emotions during gameplay. The changes in the player's emotional state according to the occurence of game events were modelled as Markov transition matrices. Next, we propose an emotion-driven adaptation algorithm (see Algorithm 2) for adapting game events according the change in the player's emotional state. The aim of adaptation is to increase positive emotions that allows involvement of the player in the game world and to avoid too much negative emotion that pushes the player to abandon the play.

Algorithm 2 An emotion-driven adaptation algorithm

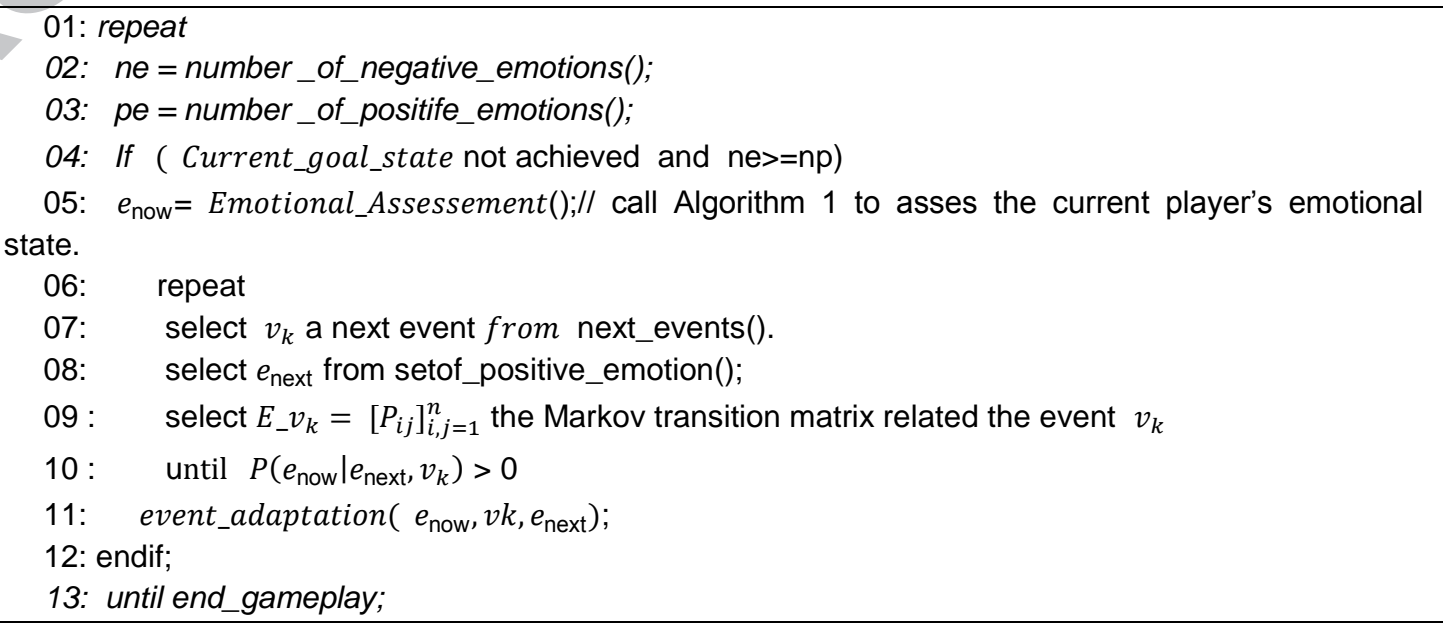


The emotion-driven adaptation algorithm is designed to perform the following operations :

- Checking the appropriate moment for performing adaptation (lines 1 to 4): It is important to determine when it becomes necessary to adapt the game upcoming events. The adaptation is not needed every time the game assesses a negative emotional state of the player because an exaggerated adaptation diminishes the perceived value of succeeding in the game (Yannakakis \& Togelius, 2011). Thus, we propose that the game adapts its events only when the number of player's positive emotion is still remains smaller than the number of player's negative emotions in a significant time interval, and the current goal is not yet reached.

- Identifing the current player's emotional state (line 5) : by invoking the emotion assessment algorithm (see Algorithm 1 in the previous section).

- Managing game events to influence the current player's emotional state (lines 6 to 12): when an adaptation is necessary, the game calls event_adaptation() a procedure that manage game events to influence the player's emotional state. This procedure may be defined by a game designer for a specific game. By regarding the transition matrices and taking into account the current emotional state, during gameplay, this procedure selects next events which can evoke positive emotion. Hence, it must be able to do smart management of next events (the upcoming events and their effects may be regulated in various ways) related to the current goal. To increase a positive emotions, this procedure orders the game mechanic to select and generate events that advance the player's progress towards achieving the current goal. So when the status of a goal is advanced, the next player's emotional state is felt as positive emotion. To avoid a negative emotion, this procedure orders the game mechanic to select and generate events that have no negative impact on the current goal. So when a goal is not at risk, the next player's emotional state remains as positive emotion. The emotion-driven adaptation algorithm will be performed at each significant time interval. Therefore, if the current goal is not achieved and the number of positive emotions is less than the number of negative emotions, then this algorithm will adapt next game events to induce positive emotions or to reduce negative ones.

\subsection{Emotions related to personality type and playing-style}

Players usually differ regarding their style of play (Hrabec, 2017) and personality types (Menekse et al., 2015; Shiota et al., 2006). Thus, in order to improve both emotion assessment and adaptation to emotions in the game playing contexts, we propose to consider personality and playing-style to personalize both emotion assessment algorithm and emotion-driven adaptation algorithm. It is well known that good games are designed in a way that allows different styles of play. Thus, players can choose different playing styles to solve problems in multiple ways (e.g. logic, systematic trial and error, free exploration, getting or not getting hints, etc.) (Tallim, 2004). Players may adopt different play styles in different games. Thus, playing styles make it possible to play the same level in different ways. A playing style presents persistent traits of the play (Bontchev \& Vassileva, 2017). Several models of playing styles, also known as player types, are proposed in the literature, like Bartle's model (Bartle, 1996), Lazzaro's model (Lazzaro, 2004, 2008), Yee's Model (Yee, 2006) and augmented Yee Model (Arelíusarson, 2013). Among these models, we focus on these associate player's emotions to playing styles. Arelíusarson (2013) proposed an augmented Yee Model, where ten emotions are mapped into player types of Yee's player types model (Yee, 2006). Lazzaro (2008) identified the most important emotional states of four player types. Thus, we suggest that the recognition of playing styles can be applied for achieving an emotion-driven personalized adaptation in the game playing contexts.

In the literature, several studies have shown that user's personality types plays a vital role in designing adaptive systems. This is because personality type "refers to those characteristics of the person that account for consistent patterns of feeling, thinking, and behaving" (Pervin, Cervone, \& Oliver, 2005). There are different personality types which can be measured using personality typing questionnaires. For example, EPQR-A personality questionnaire (Francis, Brown, \& Philipchalk, 1992) classifies individuals into three personality types. MBTI personality questionnaire classifies individuals into sixteen personality types (Myers, McCaulley, \& Most, 1985). For several years great effort has been devoted to study the relation between personality type and emotion. Thus, personality types can help to identify emotion expression patterns. For instance, Chaffar, Derbali, and Frasson (2009) found that the emotional response to a stimulus differs from one person to another according to their personality type. Considering the player's personality type for the adaptation purposes can be beneficial to provide more personalized interaction between the game and the player (Petrovica, 2017). Based on the foregoing, different player's emotional states can be categorized according to the player's personality type and the player's playing style. Consequently, this categorization of emotions can promote personalization of the previous proposed algorithms : the emotion assessment algorithm, and the emotion-driven adaptation algorithm.

\subsubsection{An personalized emotion assessment algorithm}


Algorithm 3 describe a personalized emotion assessment algorithm. Personality type and playing style can

01: $\mathrm{Pt}=$ personality_type(id_player);// retrieving from the Player profile

Ps = playing_style(id_player); // retrieving from the Player profile

Events $=\left(v_{1}, \ldots, v_{i}, \ldots . v_{n}\right)$ Emotions $=\left(e_{1}, \ldots, e_{i}, \ldots . e_{m}\right)$ goals $=\left(g_{1}, \ldots, g_{i}, \ldots . g_{p}\right)$

Actions $=\left(\mathrm{a}_{1}, \ldots, \mathrm{a}_{\mathrm{i}}, \ldots . \mathrm{a}_{\mathrm{m}}\right)$ goalstatus $=\left(\mathrm{gs}_{1}, \ldots, \mathrm{gs}_{\mathrm{i}}, \ldots, \mathrm{gs}_{\mathrm{p}}\right)$

Daclaring : Fat $\left(e_{i}\right.$, Pt, Ps $) \rightarrow\left(a t_{1}, \ldots, a_{i}, \ldots . a t_{m}\right), e_{i} \in$ Emotions,$a_{i} \in$ Actions .

Daclaring : $\mathrm{Fe}\left(\mathrm{at}_{\mathrm{i}}, \mathrm{Pt}, \mathrm{Ps}\right) \rightarrow \mathrm{Eat}_{\mathrm{i}}=\left(\mathrm{em}_{1}, \ldots, \mathrm{em}_{\mathrm{i}}, \ldots . \mathrm{e}_{\mathrm{mn}}\right), \mathrm{em}_{\mathrm{i}} \in$ Emotions

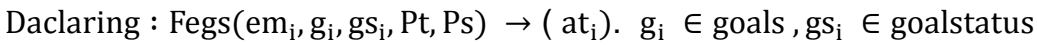

02: repaeat

03: If an event $v_{i}$ occurred then $/ / v_{i}$ is provided by the game mechanics

04: $\mathrm{g} \leftarrow$ Current_goal(); // provided by the game mechanics

05: gS $\leftarrow$ Current_goal_state(); //provided by the game mechanics

06: CA $\leftarrow$ Current_action(); // provided by the game mechanics

07: Eat $_{\mathrm{i}}=\mathrm{Fe}(\mathrm{CA}, \mathrm{Pt}, \mathrm{Ps})$;

08: For each e $\in$ Eat $_{\mathrm{i}}$ do

09: $\quad$ At $\leftarrow$ Fegs $\left(\mathrm{e}, \mathrm{g}, \mathrm{g}_{\mathrm{s}}, \mathrm{Pt}, \mathrm{Ps}\right)$;

10: $\quad$ if $($ At $=$ CA $)$ then Current_emotion $\leftarrow$ e; End_If.

13: End_For

14: end_If

15: until end_gameplay

be easily identified using appropriate questionnaires that match the specific needs of the game under examination . In that respect, the personality type and the playing style are considred as part of the player's profile when designing the personalized emotion assessment algorithm. In this algorithm the mapping functions are defined according to the same principle in Algorithm 1 (see section 4.3.3). However, these mapping functions are modified to take into account the perosnolity and the playing style of the player.

Algorithm 3 A personalized emotion assessment algorithm

\subsubsection{An emotion-driven personalized adaptation algorithm}

In the emotion-driven adaptation algorithm, transition matrices are used for tracking the change in the

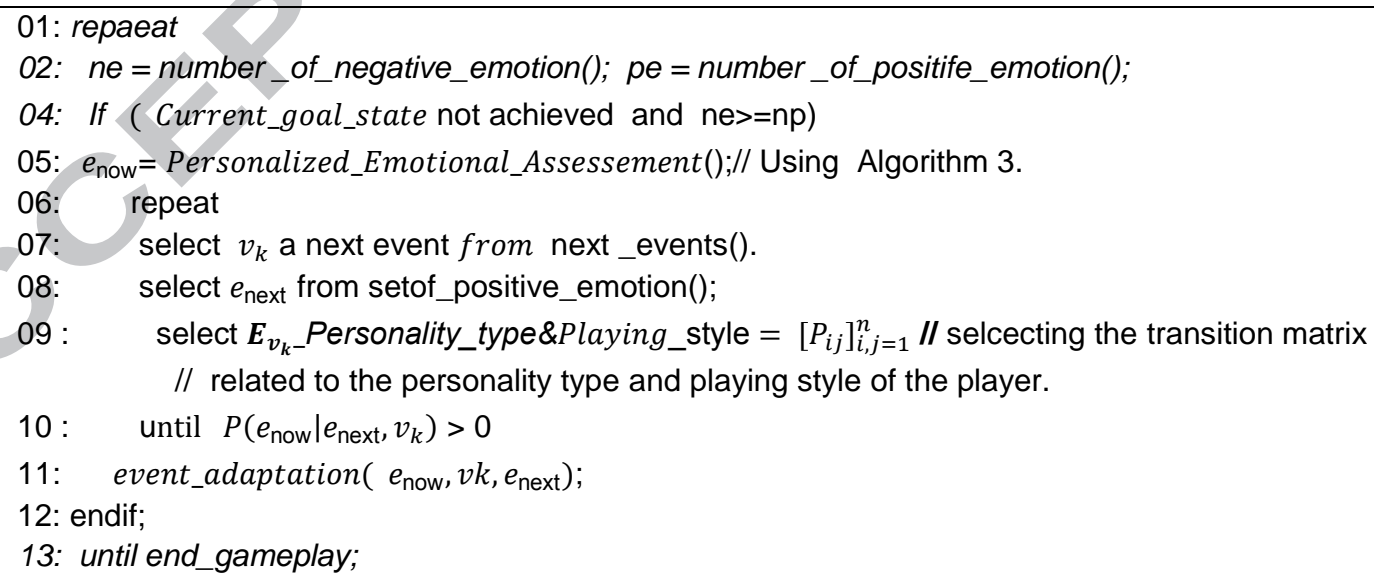

player's emotional state without considering any aspects of the player. Thus, for personalizing the emotionalbased adaptation algorithm (see Algorithm 2, in section 4.4.3), we propose a transition matrix for each possible pair of personalities types and playing styles. The obtained transition matrices describe patterns of emotional reactions in responses to each event of a particular game based on both player personality type and playing style of the player. Algorithm 4 outlines an emotion-driven personalized adaptation algorithm, where a transition matrix (The ninth line of algorithm) is selected by taking into account the personality type and playing style of the player.

Algorithm 4 An emotion-driven personalized adaptation algorithm 


\subsection{A practical methodology to develop an affective module for any serious game}

Any serious game could be adjusted to an affective version by developing the affective game module (see the architecture in section 4.2). However, the implementation of the affective module is heavily dependent on the game under examination and require some pre-experiments with the original version of the game in order to : (1) identifying the emotions that can elicited by this game, (2) establishing the mapping relation between emotion, action, goals stats, and (3) Calculating the transition probabilities matrices for game events. For this purpose, we propose a practical methodology that describes, as depicted in Fig.7, the sequential phases for implementing both the personalized emotion assessment algorithm and the emotion-driven adaptation algorithm of the affective module.

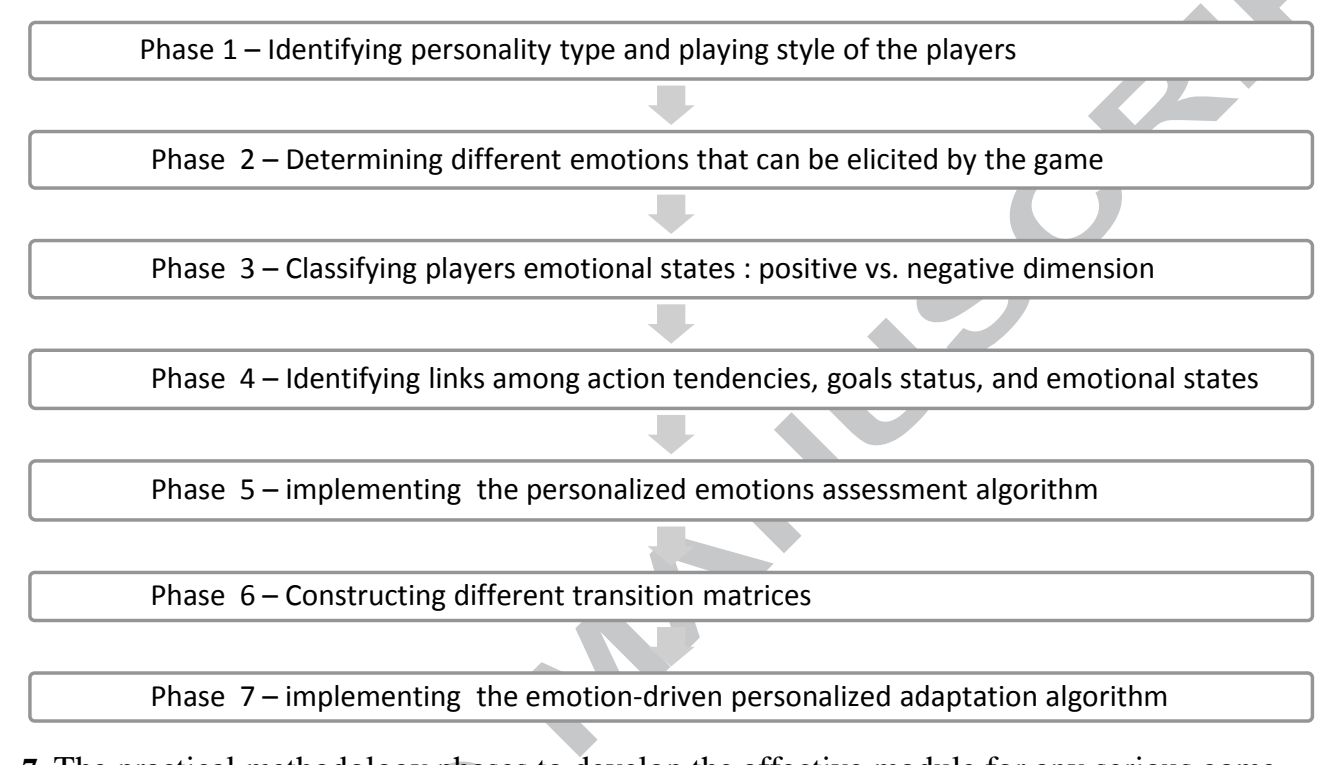

Fig. 7 The practical methodology phases to develop the affective module for any serious game.

The practical methodology spans seven sequential phases:

Phase 1- Identifying personality type and playing style of the players : as described earlier (see section 4.5), the personality type and the playing style of the player can be perfectly exploited to customise both the emotion assessment algorithm and the emotion-driven adaptation algorithm. The information about the playing style and the personality type of the player may be retrieved directly from the player profile if they already exist, otherwise any existing questionnaires from the literature can be adopted.Thus, the questionnaires must be addressed to players when creating their game accounts. For this, the questionnaires can be incorporated into the account creation interface of the game or presented separately in an external application.

Phase 2-Determining different emotions that can be elicited by the game: the aim is to identify which emotions can be elicited by both learning components and gaming components during the player-game interaction. To this end, any emotion recognition method for the self-reported data approach or physiological response can be used alone or used together for determining different emotions that can be allocated by the game. For the self-reported data approach, we suggest the use of two questionnaires. The first one is employed to measure the emotions related to the game learning content (emotion due to interaction with the learning components of the game), the second one is used to identify the emotions related to the fictional aspect of the game (emotion due to interaction with the gaming components). These questionnaires may be adapted from an existing one, or specially developed for the game under examination.

In practice, this phase required a pre-experiment with players. Then, after several playing sessions, the players will be invited to respond to the questionnaires. Thus, the gathered data will enable identifying different emotions of players and grouping emotions into different classes corresponding to each pair of playing style and personality type.

Phase 3 -Classifying players emotional states : positive vs. negative dimension : the goal of emotiondriven personalized adaptation is that the player experiences more positive emotions and less negative ones. Positive emotions are desirable emotions which have a positive influence on the player's behavier and engagement, while negative emotions are undesirable emotions which have a negative impact on them. To assign an emotion to a positive or negative dimension, the help of a game design expert may be required. The ultimate goal of adaptation is the induction of an emotion with positive dimension without too much focus on a precise 
emotional state or on the nature of the event that causes the induced emotion. In order to customise the adaptation processes of the emotion-driven personalized adaptation module, the different emotional states gathered during the previous phase, could be classified into two classes: positive dimension or negative dimension.

Phase 4 - Identifying links among action tendencies, goals status, and emotional states : as shown earlier in the personalized emotion assessment algorithm (see Algorithm 3 in section 4.5.1), the player's emotional state is assessed based on the mapping relation between different emotional states, goals status and the action tendencies. Goals status and action tendencies could be directly identified by the game mechanic. However, different player's emotional states could be measured using a retrospective emotion judgment protocol in which players annotate videos of their actions immediately after the playing session. While watching the video of their playing, players can report the emotional state they had felt most before each action they did with taking into account the current goal status. Thus, several values of (player_personality, player_type, action, goal status, emotional state) could be obtained. After that, theses values will be analysed and approved by a game design expert in order to construct an adequate data structure of the mapping relation for each pair of playing style and personality type.

Phase 5 - As a result of the previous phase, for each pair of playing style and personality type, there is a set of emotional states assigned to one of two dimensions (positive vs. negative dimension), and a data structure of the relationbetween action, goal status and emotional states. Exploiting these data, the personalized emotions assessment algorithm can be implemented in this phase.

Phase 6 - Constructing different transition matrices : As described in emotion-driven personalized adaptation algorithm (see Algorithm 4 in 4.5.2), we propose to construct, for each game event, a transition matrix that contains the probabilities to switch from one emotional state to another (where the emotion assessment algorithm, implemented earlier in phase 5, could be used to assess the emotional state). This requires a preexperiment with players, in order to construct all transition matrices related to all events of the game under examination. Within the pre-experiment, the transition probabilities can be computed by using the formula given earlier (see section 4.4.2).

Thus, the transition matrices values may be calculated during gameplay or at the end of the pre-experiment. In the first case, it can be automatically calculated during the gameplay by developing and integrating into the game a special function to compute and update the probability of transition after the occurrence of each game event. In the second case, it can be computed by exploiting the game log file to extract information about the event and the occurrence's numbers of transactions from an emotional state to another. Transition matrices can be implemented within any adequate data structure and used later in the implementation of the emotion-driven personalized adaptation algorithm.

Phase 7 - Implementing emotion-driven personalized adaptation algorithm : In this phase, we developed the emotion-driven personalized adaptation module can be implemented by exploiting the transition matrices to determe when (adaptation could be evoked when the number of negative emotional states still greater than positive ones in a certain time interval and the current goal is not yet achieved) and how (adaptation could be achieved with a smart management of the upcoming events to induce a positive emotional state or avoid a negative one) to adapt game events. Whenever the game has a substantial amount of events, the emotion-driven adaptation module will have a large amount of possibilities to manage the occurrence of upcoming events and their effects in accordance with the change in the player's emotional state. However, this requires a huge amount of training data to model the relation between all game events and the change in the player's emotional state.

\section{Experiment and Evaluation}

This section presents an experimentation with the proposed approach as well as the obtained results and their interpretation. In this sense, a specific serious game have been designed in order to following-up the practical methodology explained earlier. Particular attention has been put on the evaluation of the player's emotion as it is of utmost importance to evaluate if the proposed emotion-driven approach can affect on the change in the player's emotional states. Moreover, other measures are taken into account in order to compare and evaluate the play performance and the learning outcomes of the players.

\subsection{Experiment requirements}

The experiment required a comparison between an affective vs. non-affective version of the same serious game. Thus, in order to properly evaluate the emotion-driven approach, we conducted an experiment with two versions of a game that we have designed and developed specially for this purpose. Where, the adaptation 
strategy in the affective version consists in emotion-driven adaptation of game events, whereas the adaptation strategy in the non-affective version consists in performances-based adaptation of game levels difficulty.

The following subsections describe three versions of the same serious game: the original serious game called "Drone Against Programmed Machines" (DAPM), the affective version of DAPM and the non-affective version of DAPM.

\subsubsection{DAPM: a serious game for training on the evaluation of computer program evaluation}

DAPM is a training game (see Fig.8) that comprises six levels. The gaming missions are concerned with challenging player's knowledge and practical skills about the evaluation of simple programs written in a specific programming language (at the moment, DAPM supports the Pascal language) and requiring resource management ability in the use of weapons of a military drone. Thus, the serious goal of DAPM is to train the player to evaluate simple programs that run the enemy machine. The fun goal is to protect manufacturers by controlling a military drone to destroy the attacking enemy machine. One one hand, the military drone has a limited number of weapons (a submachine gun, missiles with different destructive forces, explosive bombs,.. etc.). On the other hand, each machine has properties such as its resistance, its destructive power, and its range of missiles. All information about a machine properties are hidden inside a control program of the attacking enemy machine. To achieve the mission's objectives for a level, the player directs the military drone and use its radar to display the program that runs the attacking machine and evaluate it in order to obtain special information values about this machine (the intensity of its resistance to weapons, the range of its shells... etc..). Then he/she uses the weapons of the military drone according to the constraints (constraints related to the use of weapons, the mission time, the protection of manufacturers, etc..) of the current mission in order to properly destroy this attacking machine.

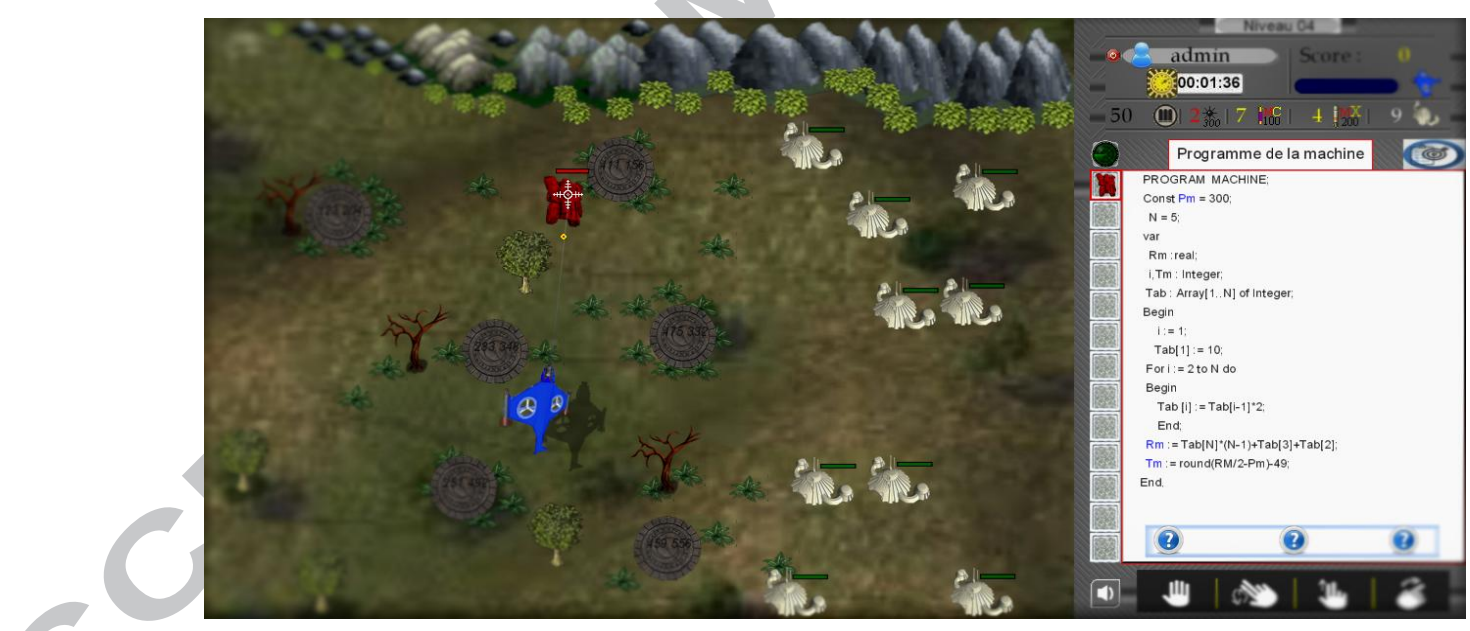

Fig. 8 A screenshot of DAPM (Drone against Programmed Machines)

The original version of DAPM is developed in a way that guarantees a progressive difficulty of the content (programs) and the challenge between levels. That is, level one is the easiest, and level six is the most difficult. Furthermore, the difficulty of the programs can be dynamically adjusted to match the degree (advanced, intermediate, junior) of the player's knowledge. This last information may be obtained from the player's profile or estimate after playing the first level.

DAPM is implemented as an online game. Where, a centralised database is used to store all the relevant information about the playing progress of each player, and also for saving data which will be used later to develop the game affective module.

\subsubsection{The affective version of DAPM: emotion-driven adaptation of game events}

In order to develop the affective version of DAPM, we followed the methodology (as described earlier in section 4.6). The methodology phases are executed sequentially (see Table 3) for developing the personalized 
emotion assessment module and the emotion-driven personalized adaptation module. These modules are embedded within the game affective module as described earleir in the architecture of affective games.

The adaptation in the affective version consists in automatically managing the occurrence of upcoming events and their effects in accordance with the change in the player's emotional state. It is well known that the player became unmotivated to keep playing if the play became boring (for very easy games) or frustrating (for very hard games). Based on that, adaptation process is activated in one of the two following baseline conditions:

- When recognizing consecutive negative emotional states within a specified time interval. Consecutive negative emotions can demotivate the player to keep playing because it can be seen as a sign that the gameplay is becoming very hard for the player. Thus, adaptation becomes necessary.

- When recognizing consecutive positive emotional states within a specified time interval. Consecutive positive emotions can demotivate the player to keep playing because it can be seen as a sign that the gameplay is becoming very easy for the player. Thus, adaptation becomes necessary.

For both proficient and non-proficient players, the adaptation , as explained earlier, would be able to monitor the change in player's emotions and maintain in real time an appropriate level of challenge. Thus, the adaptation in the affective version to a proficient vs. a non-proficient player can be explained from the quantitative aspect of the adaptation. Thus, a proficient player should be able to control the play and maintains his/her positive emotional state as much as possible. Consequently, there is less need for adaptation, which arises as reaction to significant change in the player's emotional state. In contrast, for a non-proficient player, there is more need for adaptation, since the player cannot control the play and maintain a positive emotional state.

Table 3 The phases of the practical methodology followed to develop the affective version of DAPM

\begin{tabular}{|c|c|}
\hline ase $^{\mathrm{N}^{\circ} \mathrm{ph}}$ & Description of the work done \\
\hline 01 & $\begin{array}{l}\text { - To identify the personality type of the player, we used the "Abbreviated form of the Revised Eysenck } \\
\text { Personality Questionnaire", designated by Francis et al. (1992). Therefore, based on the work of Lazzaro } \\
\text { (2008), we constructed a questionnaire to identify four playing styles. } \\
\text { - When creating their game accounts, the players are asked to answer playing style and personality } \\
\text { questionnaires which are previously implemented and integrated incorporated into the account creation } \\
\text { interface of DAPM. }\end{array}$ \\
\hline 02 & $\begin{array}{l}\text { - To measure emotions which are relate to the learning component of DAPM, an adapted version of the } \\
\text { AEQ-M questionnaire (Pekrun et al. 2005) is employed. The original AEQ-M scales assess students' } \\
\text { general emotions experienced in mathematics. However, it can be adapted to other domains as well. So, } \\
\text { we adapted accordingly the AEQ-M scales in order to assess players' emotions experienced in single } \\
\text { achievement situations when playing and dealing with programs in the context of DAPM game. } \\
\text { - To identify emotions that are relate to the gaming component of DAPM, we construct a questionnaire } \\
\text { based on Lazzaro ( } 2008,2004 \text { ), in which it has been highlighted four key that can be used to identify the } \\
\text { main player's emotions in a gaming context. } \\
\text { - In practice, forty players are invited to play DAPM and then we asked them to answer questions of the } \\
\text { abovementioned questionnaires. Thus, The gathered data enable us to identify for each class of personality } \\
\text { type and playing style different emotions that can elicited by DAPM. }\end{array}$ \\
\hline 03 & $\begin{array}{l}\text { - For each class of personality type and playing style, we classified, with the help of game expert, different } \\
\text { emotional states that are experienced by the players during gameplay, into two classes: positive dimension } \\
\text { or negative dimension. }\end{array}$ \\
\hline 04 & $\begin{array}{l}\text { - A data structure of the mapping relation is created and initialized based on several values of } \\
\text { (player_personality, player_type, action, goal status, emotional state) measured using a retrospective } \\
\text { emotion judgment protocol as described earlier in phase } 4 \text { (see section 4.6). All the mapping relation } \\
\text { values are approved by a game expert. }\end{array}$ \\
\hline 05 & $\begin{array}{l}\text { - Implementation of the personalized emotions assessment algorithm (see Algorithm } 3 \text { in section } 4.5 .1 \text { ) and } \\
\text { inetgartion it as a module as described in the affective architecture (see section 4.2) }\end{array}$ \\
\hline 06 & $\begin{array}{l}\text { - Conducting a pre-experiment, with forty university students, in order to construct all transition matrices } \\
\text { related to all events of DAPM. Probability values for each transition matrix are computed (on the basis of } \\
\text { formula given in section 4.4.2) using a computational procedure previously developed and integrated into } \\
\text { DAPM. }\end{array}$ \\
\hline 07 & $\begin{array}{l}\text { - Implementation of the emotion-driven personalized adaptation algorithm and integrate it as a module as } \\
\text { described in the affective architecture. The management events procedure is designed to provide a } \\
\text { customized adoption in accordance with the change in the player's emotional assessed by taking into } \\
\text { account his/her personality and playing style.. }\end{array}$ \\
\hline
\end{tabular}




\subsubsection{The non-affective version of DAPM: performances-based adaptation of game difficulty}

In the absence of the emotional aspect, the adaptation of a non-affective version of DAPM may be realized by adjusting, in a certain way, the gaming difficulty. Thus, the adaptation strategy of the non-affective version consists in adjusting the degree of overall difficulty (easy, normal, or hard difficulty) of the next level according to the degree of the player's play performance (low, medium or high performance) measured during the last or previous played level. Thus, a low, medium or high degree of performance (in the last played level) respectively corresponds to an easy, normal, or hard degree of difficulty (in next play level).

The difficulty baseline of a game level concerns two aspects: (1) the complexity of the program to be evaluated by the player, and (2) the setting of different gaming parameters to make the play more easy or more hard. When a game level starts, the difficulty baseline is set by considering the parameters related to the player's performance, which are measured during the previous played level.In the non-affective version, the adaptation to a proficient vs. non-proficient player concerns only the degree of difficulty of levels. Thus, a proficient player may play all levels with hard difficulty. In contrast, a non-proficient player may play all levels with easy difficulty.

\subsection{Experiment process}

Fig. 9 describe the steps of the experiment process where a control and experimental group of players respectively played a non affective and an affective version of DAPM. The aim is to study whether the affective version would improve the player's emotional experience and performance compared to the non-affective

\begin{tabular}{|l|l|}
\hline 1 & $\begin{array}{l}\text { • Creating game accounts } \\
\text { - Taking questionnaires: Personality/ playing styles }\end{array}$ \\
\hline 2 & • Performing a trial session to familiarise with DAPM controls. \\
\hline 3 & $\begin{array}{l}\text { • Pretest } \\
\text { - Assigning players to control or experimental group }\end{array}$ \\
\hline 4 & - Performing several proper playing sessions \\
\hline 5 & - Taking the Immersive Experience Questionnaire (IEQ) (Jennett \\
\hline 6 & et al. 2008) \\
\hline 6
\end{tabular}

version.

Fig. 9 General scheme (steps) of the experiment process.

\subsubsection{Participants}

The experiment is conducted with control vs. an experimental group where each group is composed of 15 university students, 6 female players and 9 males, between 19 and 28 years old. No player has experience with the game used in this study. In each group, ten participants played video or serious games very rarely, and the rest at least play games several times a week. More details about the criteria followed to assign players to thier groups is given later ( see step 3, in section 5.2.3).

\subsubsection{Materials}

The affective and the non- affective versions of DAPM are implemented as web game. For each version, a centralized database was used to save the values of relevant play indicators for each player, such as scores, playing time, the frequency of positive / negative emotions, number of failed attempts before completing a given level,... etc. To identify the playing style and personality type of the player, the same questionnaires adopted in the development of the game affective module are integrated into the player's account creation interface. Thus, personality type and playing style are considered as part of the player profile.

To measure the overall emotional involvement of players whilst playing, the Immersive Experience Questionnaire (IEQ) (Jennett et al., 2008) was used. The IEQ is a broadly applicable questionnaire, in different game types, for determining the level of player's immersion. Immersion is considered as a single dimension compound of five factors: cognitive involvement, real world dissociation, challenge, emotional involvement and 
control. The IEQ was implemented as a web survey. For the pretest and posttest, we used ten simple exercises about the evaluation of certain Pascal programs such that each exercise is graded out of ten points.

\subsubsection{Procedure steps}

To achieve the experimental process, the following procedure steps were applied :

Step 1: After the description of the purpose and procedure of the experiment, all players are invited to create their own game accounts. At the end of registration, players were invited to complete the personality type questionnaire and the playing style questionnaire.

Step 2 : In this step, each player was allowed to try out the game in a trial session in order to familiarise themselves with DAPM controls. There are no restrictions in time and the score was not recorded, and the players were allowed to stop whenever they thought they were ready for the proper gaming session.

Step 3 : After taking a pretest about Pascal programming exercises evaluation, players are separated into two groups (experimental vs. control group ) in such a way that the average pretest score of the experimental group was either equal or convergent to the average pretest score of the control group. In order to make a balance in terms of the number of players who have experience playing video games, players with equal scores are transferred between the two groups based on their general experiences in playing video games. The aim is to create two equal groups in terms of the players' their knowledge relating to serious goal of DAPM and also in terms of their general gaming experience.

Step 4 : Players of the control group are asked to play the non-affective version of DAPM. Players of the experimental group are requested to play the affective one. All players of both groups are invited to play and try to complete the game for up to two hours. For a successful mission, a player may be obliged to replay the same level several times before progressing to the next level. Thus, the player's progress indicators were automatically stored in the DAPM database.

Step 5 : When all playing sessions end, each player is invited to access the web survey page of IEQ. The players' scores of IEQ are saved in the DAPM database. The IEQ uses five-point Likert scale questions for measuring the player experience; its overall score is composed of the summary of the results from questions of its five factors.

Step 6 : In this phase, a posttest was administered to compare players' improvement regarding the evaluation of pascal programs after playing DAPM.

At the end of the experiment, different results are obtained: playing indicators results, IEQ scores results, and the postest results, which will be presented and discussed in the following sections.

\subsection{Results}

\subsubsection{Playing indicators results}

During the experiment process, players are invited to complete in order the missions of six levels with progressive difficulties. The player must respect all the constraints of a mission in order to achieve a level, otherwise he/she will stay (repeat) the same level several times. The duration of a level is between two and five minutes. At the beginning of each level, all values of game indicators are reinitialized. Table 4 represents these indicators and their designation. The average measurement results of these indicators, for all players in each group, are summarized in Table 5. We distinguished between the indicators of play like the score, and other indicators of evaluation for to compare the emotional aspect across players. Indicators are recorded only for the first time the player successfully ends the mission of a level. An explanation is given below in order to understand indicator results

Table 4 Indicators and their designation

\begin{tabular}{|l|l|}
\hline Indicator & Designation \\
\hline NA & The number of the player's Negative Attempts in playing to gain a level. \\
\hline PE & The number of the player's Positive Emotional state assessed at each gained level. \\
\hline NE & The number of the player's Negative Emotional state assessed at each gained level. \\
\hline SC & The player's SCore at each gained level. \\
\hline
\end{tabular}

Table 5 The average measurement results

\begin{tabular}{|c|c|c|c|c||c|c|c|c|}
\cline { 2 - 9 } \multicolumn{1}{c|}{} & \multicolumn{4}{c||}{ Experimental group } & \multicolumn{4}{c|}{ Control group } \\
\cline { 2 - 9 } \multicolumn{1}{c|}{} & \multicolumn{4}{c||}{ The average value of } & \multicolumn{4}{c|}{ The average value of } \\
\cline { 2 - 9 } & NA & SC & PE & NE & NA & SC & PE & NE \\
\hline Level 1 & 01 & 700 & 40 & 38 & 02 & 700 & 34 & 33 \\
\hline Level 2 & 02 & 700 & 40 & 37 & 03 & 650 & 35 & 35 \\
\hline
\end{tabular}




\begin{tabular}{|c|c|c|c|c||c|c|c|c|}
\hline Level 3 & 02 & 650 & 44 & 34 & 03 & 600 & 35 & 40 \\
\hline Level 4 & 02 & 700 & 42 & 32 & 04 & 450 & 27 & 32 \\
\hline Level 5 & 02 & 750 & 46 & 30 & 04 & 400 & 24 & 45 \\
\hline Level 6 & 03 & 800 & 54 & 28 & 06 & 350 & 29 & 51 \\
\hline \hline $\begin{array}{c}\text { The overall } \\
\text { average value }\end{array}$ & $\mathbf{2}$ & $\mathbf{7 1 0 . 6 6}$ & $\mathbf{4 4 . 3 3}$ & $\mathbf{3 1 . 1 6}$ & $\mathbf{4}$ & $\mathbf{5 2 0 . 5 0}$ & $\mathbf{3 0 . 6 6}$ & $\mathbf{3 9 . 3 3}$ \\
\hline
\end{tabular}

NA indicator: for NA indicator, its overall average value varies between two and six. In control group, the average value of NE indicator was increasing from level to another. It can also be observed that the NA average values in control group is double of that in experimental group.

SC indicator: the SC indicator is used as a measurement of playing performance. As seen in Fig. 10, the average SC values of the control group are lower than of the experimental group. In control group, average SC values are decreasing and inversely proportional to the progress in level of play. In experimental group, despite the increasing difficulty, average SC values are increasing from level one to level six.

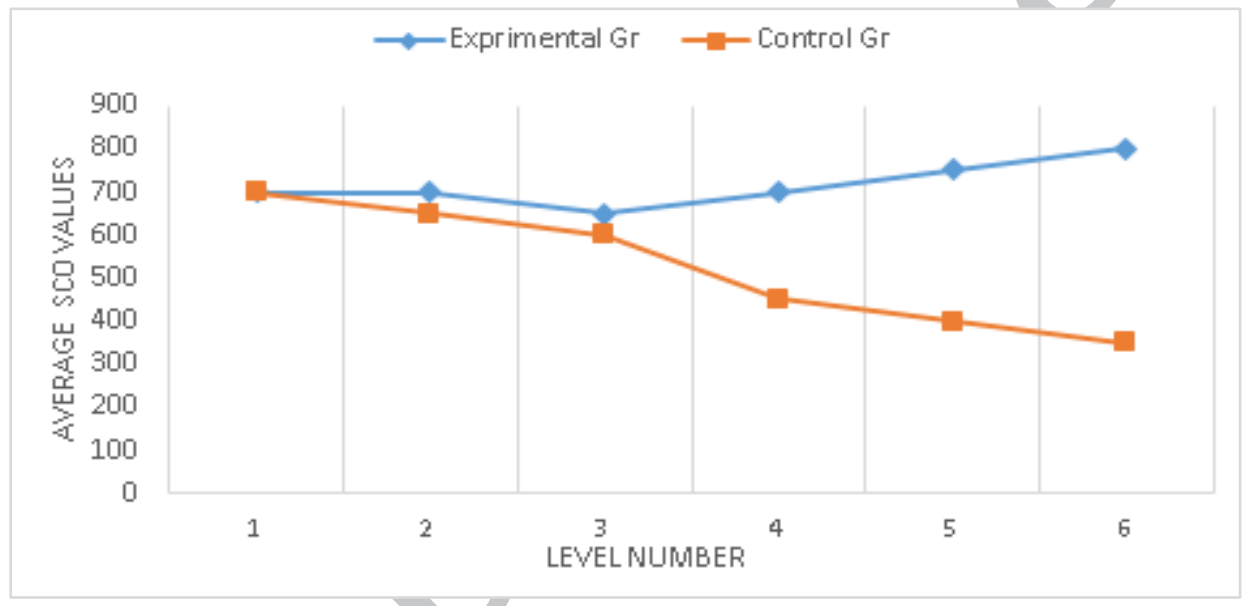

Fig. 10 Variation of average scores (SC) in the six levels of DAMP

PE and NE indicators : for each gained level, the number of emotional states assessed during gameplay is calculated. PE indicator designates the number of positive emotional states. NE indicator designates the number of negative emotional states. As can be seen in Table 5, the average PE/NE values in the experimental group are generally higher than these in the control group.

As shown in Fig. 11, we observe an increase of PE percentages from level one to level six in the experimental group. Conversely, a decrease of PE percentages from level one to level six is observed in the control group.

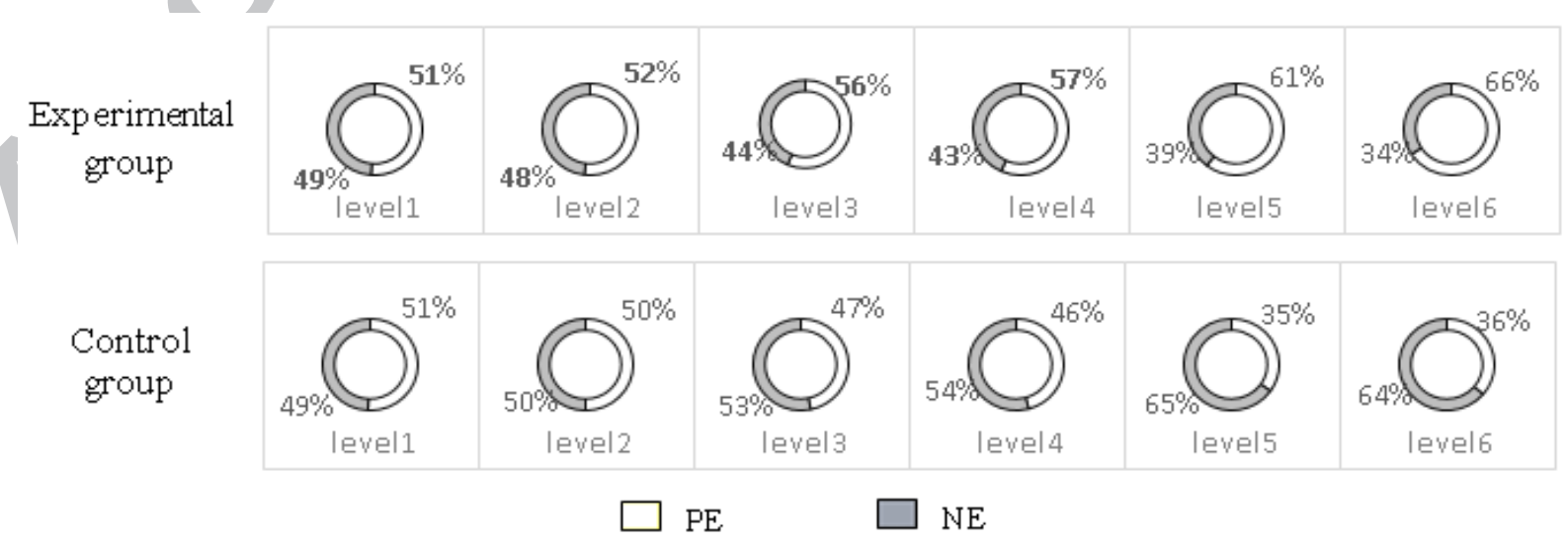

Fig. 11 Percentage of PE/NE in each level for both groups

\subsubsection{IEQ results}

To map the IEQ scores of all players in each group, we used a Box-plots, that enable us to study the distributional characteristics of the level of IEQ scores in each group. To construct a Box Plot graph (see Fig. 
12), IEQ scores are sorted. Then four equal sized parts are made from the ordered scores. We label these parts one to four, starting at the left. That is, $25 \%$ of all scores are placed in each part. The vertical line that divides each box into two parts, called the median, marks the mid-value of the IEQ scores. The box plot of the experimental group is higher than the equivalent plot of the control group. Thus, in experimental group, the median scores are greater than or equal to 135 and 25\% of players have scores between 135.19 and 145 . While in control group, the median scores are greater than or equal to 116.45 and $75 \%$ of players have scores between 80 and 127.

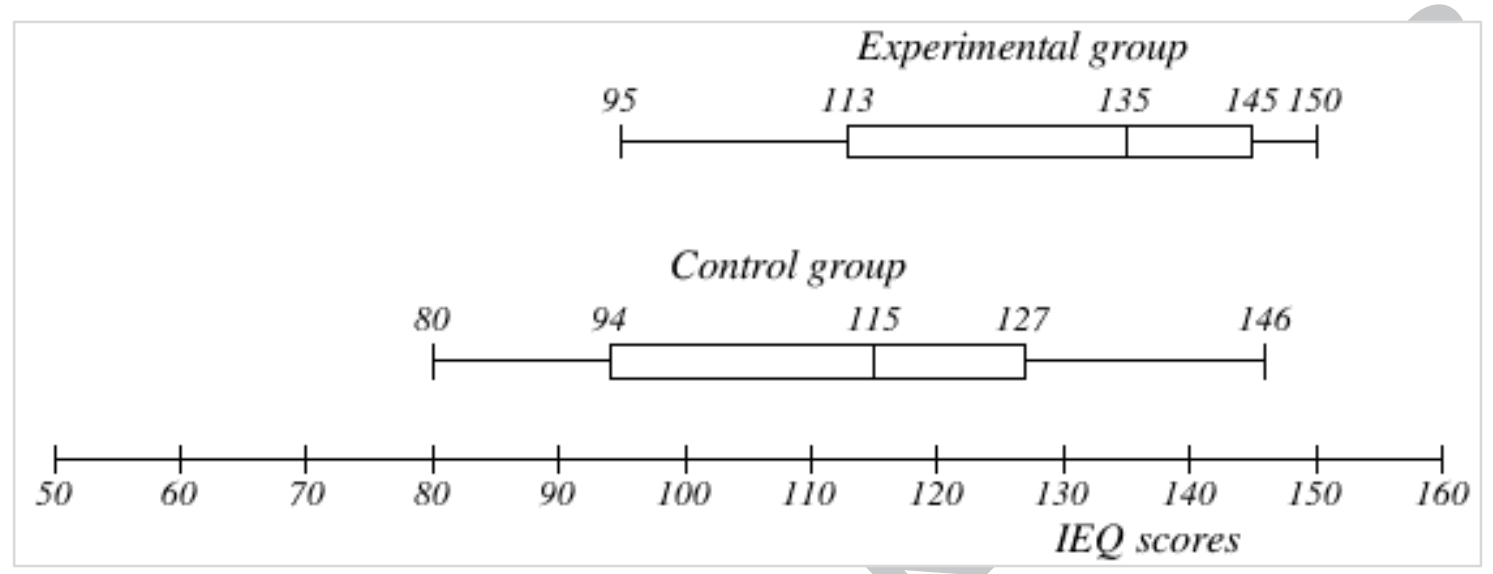

Fig. 12 The boxplot of IEQ scores of players in experimental /control group.

Table 6, shows overall average scores of IEQ and its five factors for each group. There was a significant difference in emotional involvement, cognitive involvement and control components. There was no significant difference in the real world dissociation and challenge components. In general, there was a difference of 20.00 scoring units on the IEQ scale, between the overall average scores of the two groups. Thus, It seems that players who played the affective version are more immersed than ones played the non-affective version. In terms of emotional involvement component, there was a significant difference in IEQ average scores between the two groups. The items used for calculating the emotional involvement component are questions expected to measure emotion related to the game events.

Table 6. overall average scores of IEQ and its components in control and experimental groups.

\begin{tabular}{|c|c|c|c|}
\hline & & \multicolumn{2}{|c|}{ The average scores of } \\
\hline & & $\begin{array}{l}\text { Experimental group } \\
\text { (affective DAPM) }\end{array}$ & $\begin{array}{c}\text { Control group } \\
\text { (Non- affective DAPM) }\end{array}$ \\
\hline \multirow{5}{*}{ 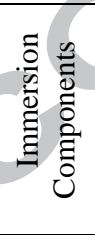 } & emotional involvement & 31.00 & 20.00 \\
\hline & cognitive involvement & 39.50 & 37.10 \\
\hline & real world dissociation, & 24.10 & 22.00 \\
\hline & challenge & 16.70 & 15.20 \\
\hline & Control & 23.70 & 20.80 \\
\hline & IEQ & 135.00 & 115.00 \\
\hline
\end{tabular}

\subsubsection{Posttest results}

Table 7 shows the results of the posttest. It presents the number of players in the groups according to the following statements: Good, Middle, and Weak.

Table 7. Players' posttest results

\begin{tabular}{|c|c|c|c|}
\cline { 2 - 4 } \multicolumn{1}{c|}{} & \multicolumn{3}{|c|}{ Results : scores' range } \\
\cline { 2 - 4 } & $\begin{array}{c}{[100,6} \\
00]\end{array}$ & $\begin{array}{c}\text { ]60,30 } \\
0[\end{array}$ & {$[30,0]$} \\
\cline { 2 - 4 } & Good & Middle & Weak \\
\hline Control group & 5 & 3 & $7(47 \%)$ \\
\hline
\end{tabular}




\begin{tabular}{|l|c|c|c|}
\hline & $(33 \%)$ & $(20 \%)$ & \\
\hline Experimental group & $\begin{array}{c}9(60 \% \\
)\end{array}$ & $\begin{array}{c}4 \\
(27 \%)\end{array}$ & $2(13 \%)$ \\
\hline
\end{tabular}

Most of players, in the experimental group, have obtained good or middle results, while in the control group fewer players received the same rating. On the contrary, the number of players with weak rating in the experimental group is less than in the control group. By comparing posttest results, we can see that the most significant differences between groups are shown for good and weak rating. The most of players in the experimental group had good rating result compared to those in the control group. Conversely, most of players in control group had weak rating result.

\subsection{Discussion}

The experiment results given earlier proved that the affective version was able to improve more the emotional experience, the playing performance and the learning outcomes than the non-affective version. These results can be explained by the qualitative and the quantitative aspects of adaptation. Regarding the qualitative aspect, the adaptation in the affective version has a good impact on player's engagement because it has been done just in time during gameplay. Additionally, the adaptation in the affective version was better targeted and more efficient than in the non-affective version. That is because the emotion-driven adaptation module has been developed and customized by taking into account the personality and playing style of the player. In the inverse, the adaptation of the non-affective version has low impact on player's engagement because it has done in next time of the play (replay the same level or play the next level) and without taking into account any characteristic of the player. Regarding the quantitative aspect, the adaptation in the affective version may be done many times during gameplay. In the inverse, the adaptation of the non-affective version has been done only one time (adapting of the whole level) before starting the next play.

In the following subsections, we separately discuss in detail the implications for each of the results given earlier.

\subsubsection{Discussion of playing indicators results}

As shown in Fig. 13, for the same level in the experimental and control group, when the positive emotions overshadow the negative ones (average PE values are higher or equal to average NE value) then the players' average scores (value of SC) are higher or equal to the median. Furthermore, we observe that all average values of PE indicator in the experimental group are higher than in the control group. However, due to the lack of emotional adaptation, average values of NE indicator in the experimental group are lower than in the control group. Generally, we observe that in the first level of the game, all players of both groups have experienced positive emotions more than negative ones, as well as their scores were higher than the median. This is due to the players' motivation at the beginning of play and to the easiness of the first level.

When the game's difficulty increased steadily from level one to level six, the players' positive emotions, in control group, begin to diminish in contrast to the player's negative emotions that were growing, this has led to a decline in player's motivation and thus their scores fell below the average. On the other hand, players in the experimental group have experienced more positive emotions than negative ones. In fact, the affective module enables the assessment of the player's emotional state in real time and then adapt next events accordingly to induce more positive emotions. Thus, the adaptation of DAPM events in each level were following the change in the player's emotional state in real time. Therefore, emotions-driven adaptation has led to an increase in the number of occurrences of player's positive emotions (PE) and has led to decrease the number of occurrences of negative emotions (NE). In this manner, the players did their best to get the highest possible score in each level. 


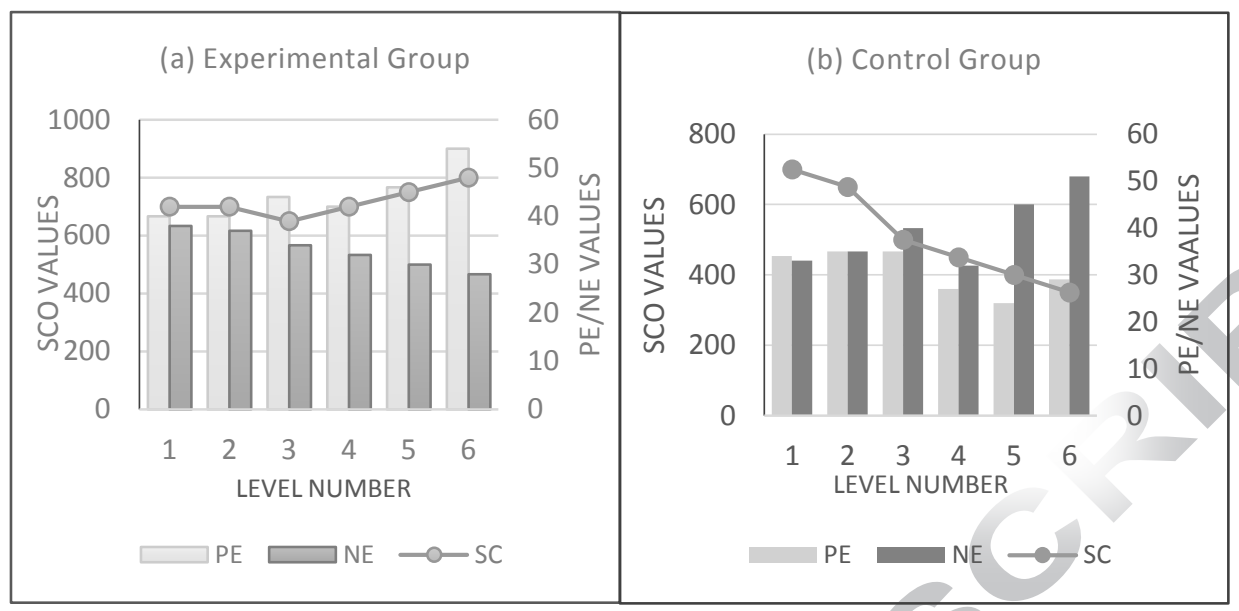

Fig. 13 Relationbetween the averages values of SC, PE and NE in the six levels of DAPM

\subsubsection{Discussion of IEQ results}

The IEQ results presented above prove that the affective version of DAPM promotes more emotional engagement and involvement than the non-affective version of DAPM. In terms of emotional involvement component, there was a significant difference in the average IEQ scores between the two groups. In order to compare in details the players' emotional involvement between the groups, a boxplot of emotional involvement scores are designed. As seen from Fig.14, the boxplot of emotional involvement scores with and without affective game shows very different distributions of scores between groups.

First, in the experimental group, players' involvement and engagement have higher average values (mean value equal to 31), while it have law average values (mean value equal to 20) in the control group. Second, in the adaptive game, $75 \%$ of players, in the experimental group, have scores varies above the overall average score, but in control group, $75 \%$ of players have scores below the overall average score. Furthermore, emotional involvement scores for the experimental group prove that the affective version invokes a high number of positive emotional states by managing the game events according to the change in the player's emotional state during gameplay. While, in the non-adaptive game, the increase of game difficulty, with the absence of adaptation, invokes a high number of negative emotional states.

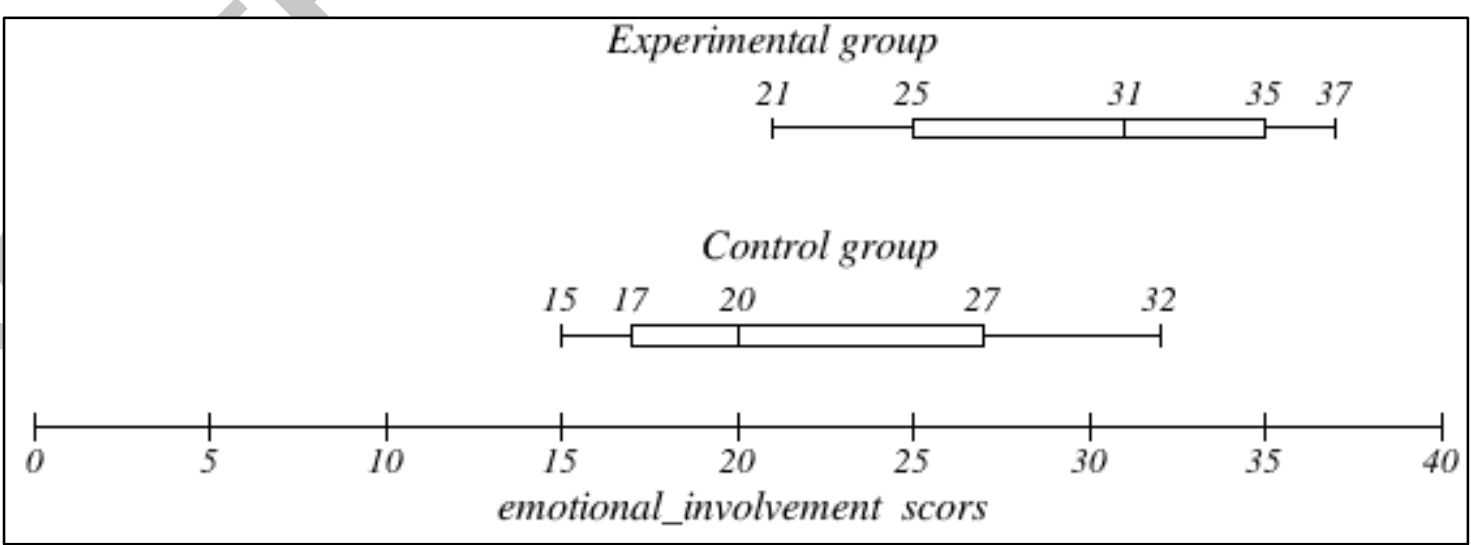

Fig. 14 The boxplot of emotional involvement scores of players

\subsubsection{Discussion of post-test results}


At the beginning of the experiment, players are separated into two similar groups (experimental vs. control group) to play two different version (affective vs. non- affective) of DAPM. In both groups, players have similar characteristics in terms of their knowledge related to the educational goal of DAPM and also in terms of their general gaming experience. In both versions of DAPM, the score for a level is computed by summing the score related to skills of playing and the score related to the mastery of knowledge. That is, in each mission of a level, if the player chooses the right weapon resources after evaluating a program of the attacked machine, then he/she will get the score related the mastery of knowledge, afterwards, if the player demonstrates mastery of skills when using weapon resources to achiev game goals of a mission, then he/she will gain score of playing skills.
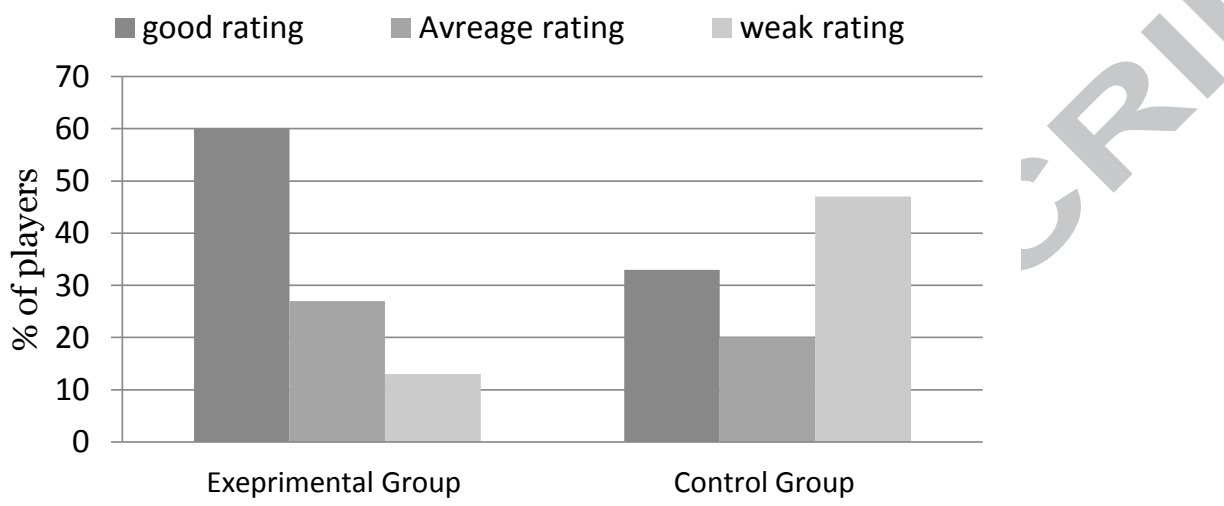

Fig. 15 Comparing posttest results: experimental vs. control group.

At the end of the experiment, players of both groups took the posttest to compare the knowledge acquisition after playing. We observed, as shown in Fig. 15, a most significant differences of result rating between groups. Most of the players in the experimental group have good rating result. Conversely, most of players in the control group have weak rating result. The number of players who obtained average results in the experimental group is higher than that in the control group. We conclude that emotion-driven personalized adaptation improved the knowledge acquisition. This is due to the fact that players of the experimental group experienced a variety of positive emotions which influenced their performance in terms of knowledge acquisition and playing skills.

\section{Conclusion and future work}

In this paper, we have proposed a new emotion-driven approach for personalized the emotion assessment and the adaptations to emotions in the serious game playing context. Thus, we have introduced, according to our view, a generic definition and architecture of affective games. Afterwards, we have proposed two methods and their algorithms: (1) the personalized emotion assessment method/algorithm, and (2) the emotion-driven personalized adaptation method/ algorithm. Where, the personalization aspect indicates that emotions are assessed taking into account the personality type and the playing style of the player. The first method focuses on assessing the player's emotional state in real time during gameplay. Thus, the personalized emotion assessment algorithm is realized by investigating the mapping relation between different player's emotional states and the game goals status on one side and the player's actions tendencies on the other side. The second method highlights a personalized adaptation of game events according to the change in the player's emotional state during gameplay. Based on a Markov modeling of the change in the player's emotional state in accordance of game events, we have proposed an emotion-driven personalized adaptation algorithm. This algorithm exploits Markov transition matrices to adapt and manage game events in order to induce positive emotions and/ or avoid negative ones. Furthermore, to demonstrate the applicability of the proposed approach; we have defined seven phases for a practical methodology for developing the game affective module for any serious game.

To validate and evaluate the emotion-driven approach, we have conducted an experiment using two versions (affective vs. non-affective) of the same game that we have designed and developed for a better translate the actual player experience. The obtained results have demonstrated the efficiency of the game affective module to increase the playing outcomes and to enhance the overall game playability.

For further evaluation and improvement of the emotion-driven approach, four major routes may be followed in future work:

- Examining adaptation quality provided by the emotion-driven approach by separately evaluating the gaming outcomes and the learning outcomes.

- Investigating the impact of the emotion-driven approach on multiplayer game adaptation. 
- proposing a semi-supervised learning method for collecting and analyzing player's gameplay data in order to continuously update, after each time a game is played, the data structures of mapping functions used in both assessment and adaptation algorithms.

- Considering the emotional intelligence of the players to improve the emotion-driven personalized adaptation. For example, the Trait Emotional Intelligence Questionnaire-Short Form (TEIQue-SF) (Petrides, 2009; Siegling, Vesely, Petrides, \& Saklofske, 2015) can be used to measure the scores of emotional intelligence of the player .

\section{References}

Abt, C. C. (1987). Serious games: University press of America.

Anolli, L., Mantovani, F., Confalonieri, L., Ascolese, A., \& Peveri, L. (2010). Emotions in serious games: From experience to assessment. International Journal of Emerging Technologies in Learning (iJET), 5(SI3), 7-16.

Aranha, R. V., Silva, L. S., Chaim, M. L., \& dos Santos Nunes, F. d. L. (2017). Using Affective Computing to Automatically Adapt Serious Games for Rehabilitation. Paper presented at the Computer-Based Medical Systems (CBMS), 2017 IEEE 30th International Symposium on.

Arelíusarson, A. (2013). Designing Computer Games Preemptively For Emotions And Player Types. Retrieved from http://gamasutra.com/blogs/AreliusAreliusarson/20130619/194574/Designing_ComputerGames_Preemptivel y_for_Emotions_and_Player_Types.php Accessed 05 April 2018.

Bartle, R. (1996). Hearts, clubs, diamonds, spades: Players who suit MUDs. Journal of MUD research, 1(1), 19.

Bianchi-Berthouze, N. (2013). Understanding the role of body movement in player engagement. HumanComputer Interaction, 28(1), 40-75.

Bontchev, B. (2016a). Adaptation in affective video games: A literature review. Cybernetics and Information Technologies, 16(3), 3-34.

Bontchev, B. (2016b). Holistic player modeling for controling adaptation in video games. Paper presented at the Proc. of 14th Int. Conf. e-Society, Vilamoura, Portugal.

Bontchev, B., \& Vassileva, D. (2017). Affect-based adaptation of an applied video game for educational purposes. Interactive Technology and Smart Education, 14(1), 31-49.

Bopp, M. M. (2008). Storytelling and motivation in serious games. Part of the Final Consolidated Research Report of the Enhanced Learning Experience and Knowledge Transfer-Project.

Brich, J., Rogers, K., Frommel, J., Weidhaas, M., Bruckner, A., Mirabile, S., . . . Weber, M. (2015). LiverDefense: using a tower defense game as a customisable research tool. Paper presented at the Games and Virtual Worlds for Serious Applications (VS-Games), 2015 7th International Conference on.

Brisson, A., Pereira, G., Prada, R., Paiva, A., Louchart, S., Suttie, N., . . . Bellotti, F. (2012). Artificial intelligence and personalization opportunities for serious games. Paper presented at the Proc. of 8th AIIDE Conf.

Cacioppo, J. T., Berntson, G. G., Larsen, J. T., Poehlmann, K. M., \& Ito, T. A. (2000). The psychophysiology of emotion. Handbook of emotions, 2, 173-191.

Chaffar, S., Derbali, L., \& Frasson, C. (2009). Inducing positive emotional state in Intelligent Tutoring Systems. Paper presented at the AIED.

Chanel, G., Rebetez, C., Bétrancourt, M., \& Pun, T. (2011). Emotion assessment from physiological signals for adaptation of game difficulty. IEEE Transactions on Systems, Man, and Cybernetics-Part A: Systems and Humans, 41(6), 1052-1063.

Connolly, T. M. (2013). Psychology, pedagogy, and assessment in serious games: IGI Global.

Connolly, T. M., Boyle, E. A., MacArthur, E., Hainey, T., \& Boyle, J. M. (2012). A systematic literature review of empirical evidence on computer games and serious games. Computers \& Education, 59(2), 661-686.

Damasio, A. R., \& Sutherland, S. (1994). Descartes' error: Emotion, reason and the human brain. Nature, 372(6503), 287-287.

Dolar, D. P. (2014). Experiments on flow and learning in games. Ph. D. thesis,

Drachen, A., Nacke, L. E., Yannakakis, G., \& Pedersen, A. L. (2010). Correlation between heart rate, electrodermal activity and player experience in first-person shooter games. Paper presented at the Proceedings of the 5th ACM SIGGRAPH Symposium on Video Games.

Egan, D., Brennan, S., Barrett, J., Qiao, Y., Timmerer, C., \& Murray, N. (2016). An evaluation of Heart Rate and ElectroDermal Activity as an objective QoE evaluation method for immersive virtual reality environments. Paper presented at the Quality of Multimedia Experience (QoMEX), 2016 Eighth International Conference on. 
Fairclough, S., \& Gilleade, K. (2012). Construction of the biocybernetic loop: a case study. Paper presented at the Proceedings of the 14th ACM international conference on Multimodal interaction.

Fink, G. A. (2014). Markov models for pattern recognition: from theory to applications: Springer Science \& Business Media.

Francis, L. J., Brown, L. B., \& Philipchalk, R. (1992). The development of an abbreviated form of the Revised Eysenck Personality Questionnaire (EPQR-A): Its use among students in England, Canada, the USA and Australia. Personality and individual differences, 13(4), 443-449.

Frijda, N. H. (1986). The emotions: Cambridge University Press.

Frijda, N. H. (1987). Emotion, cognitive structure, and action tendency. Cognition and emotion, 1(2), 115-143.

Frijda, N. H. (2007). The laws of emotion. Mawwah. In: NJ: Erlbaum.

Frommel, J., Rogers, K., Brich, J., Besserer, D., Bradatsch, L., Ortinau, I., Weber, M. (2015). Integrated questionnaires: Maintaining presence in game environments for self-reported data acquisition. Paper presented at the Proceedings of the 2015 Annual Symposium on Computer-Human Interaction in Play.

Frutos-Pascual, M., \& Zapirain, B. G. (2017). Review of the use of AI techniques in serious games: Decision making and machine learning. IEEE Transactions on Computational Intelligence and AI in Games, 9(2), 133-152.

Gasselseder, H.-P. (2014). Those who played were listening to the music? Immersion and dynamic music in the ludonarrative. Paper presented at the Cognitive Information Processing (CIP), 2014 4th International Workshop on.

Grappiolo, C., Cheong, Y.-G., Togelius, J., Khaled, R., \& Yannakakis, G. N. (2011). Towards player adaptivity in a serious game for conflict resolution. Paper presented at the Games and Virtual Worlds for Serious Applications (VS-GAMES), 2011 Third International Conference on.

Grigore, O., Gavat, I., Cotescu, M., \& Grigore, C. (2008). Stochastic algorithms for adaptive lighting control using psycho-physiological features. International Journal of Biology and Biomedical Engineering, 2, 9-18.

Hastings, E. J., Guha, R. K., \& Stanley, K. O. (2009). Evolving content in the galactic arms race video game. Paper presented at the Computational Intelligence and Games, 2009. CIG 2009. IEEE Symposium on.

Hazlett, R. L. (2006). Measuring emotional valence during interactive experiences: boys at video game play. Paper presented at the Proceedings of the SIGCHI conference on Human Factors in computing systems.

Hocine, N., \& Gouaich, A. (2011). A survey of agent programming and adaptive serious games.

Holm, S. K. (2017). Player Types: What, Why and How. Paper presented at the Extended Abstracts Publication of the Annual Symposium on Computer-Human Interaction in Play.

Hoskin, R. (2012). The dangers of self-report-Science Brainwaves. In.

Hrabec, O. (2017). Categorizing Play Styles in Competitive Gaming. International Journal of Gaming and Computer-Mediated Simulations (IJGCMS), 9(4), 62-88.

Hudlicka, E. (2008). Affective computing for game design. Paper presented at the Proceedings of the 4th Intl. North American Conference on Intelligent Games and Simulation.

Hudlicka, E. (2009). Affective game engines: motivation and requirements. Paper presented at the Proceedings of the 4th international conference on foundations of digital games.

Ismailović, D., Haladjian, J., Köhler, B., Pagano, D., \& Brügge, B. (2012). Adaptive serious game development. Paper presented at the Games and Software Engineering (GAS), 2012 2nd International Workshop on.

Jennett, C., Cox, A. L., Cairns, P., Dhoparee, S., Epps, A., Tijs, T., \& Walton, A. (2008). Measuring and defining the experience of immersion in games. International journal of human-computer studies, 66(9), 641-661.

Karpouzis, K., \& Yannakakis, G. N. (2016). Emotion in Games: Springer.

Karpouzis, K., Yannakakis, G. N., Shaker, N., \& Asteriadis, S. (2015). The Platformer Experience Dataset. Paper presented at the 6 th international conference on affective computing and intelligent interaction.

Karpouzis, K., Yannakakis, G. N., Paiva, A., Nielsen, J. H., Vasalou, A., \& Jhala, A. (2013). User Modelling and Adaptive, Natural Interaction for Conflict Resolution. 2013 IEEE Humaine Association Conference on Affective Computing and Intelligent Interaction, 719-721.

Kaye, L. K., \& Bryce, J. (2012). Putting the fun factor into gaming: The influence of social contexts on the experiences of playing videogames. International Journal of Internet Science, 7(1), 24-38.

Kickmeier- Rust, M. D., \& Albert, D. (2010). Micro- adaptivity: Protecting immersion in didactically adaptive digital educational games. Journal of Computer Assisted Learning, 26(2), 95-105.

Kivikangas, J. M., Chanel, G., Cowley, B., Ekman, I., Salminen, M., Järvelä, S., \& Ravaja, N. (2011). A review of the use of psychophysiological methods in game research. journal of gaming \& virtual worlds, 3(3), 181-199.

Kleinsmith, A., \& Bianchi-Berthouze, N. (2013). Affective body expression perception and recognition: A survey. IEEE Transactions on Affective Computing, 4(1), 15-33. 
Lankoski, P. (2012). Computer games and emotions. In The Philosophy of Computer Games (pp. 39-55): Springer.

LARSEN, J. T., BERnTSON, G. G., POEHLMANN, K. M., ITO, T. A., \& CACIOPPO, J. T. (2010). The Psychophysiology of Emotion. Handbook of emotions, 180.

Lazzaro, N. (2004). Why we play games: Four keys to more emotion without story.

Lazzaro, N. (2008). The Four Fun Key. GU Experience. In: Morgan Kaufmann.

Liu, C., Agrawal, P., Sarkar, N., \& Chen, S. (2009). Dynamic difficulty adjustment in computer games through real-time anxiety-based affective feedback. International Journal of Human-Computer Interaction, 25(6), 506-529.

McMahan, T., Parberry, I., \& Parsons, T. D. (2015). Modality specific assessment of video game player's experience using the Emotiv. Entertainment Computing, 7, 1-6.

Medler, B. (2011). Player dossiers: Analyzing gameplay data as a reward. Game Studies, 11(1).

Mena, R. J. R. (2012). Player types, play styles, and play complexity: Updating the entertainment grid. International Journal of Game-Based Learning (IJGBL), 2(2), 75-89.

Menekse, G., Çagiltay, N. E., \& Ozcelik, E. (2015). Personality type indicator models in serious games: A case study in a surgical navigation game. Paper presented at the Information Technology Based Higher Education and Training (ITHET), 2015 International Conference on.

Mesquita, B. (2016). The legacy of Nico H. Frijda (1927-2015). In: Taylor \& Francis.

Myers, I. B., McCaulley, M. H., \& Most, R. (1985). Manual, a guide to the development and use of the MyersBriggs type indicator: Consulting Psychologists Press.

Noldus. (2014). FaceReader: Tool for Automated Analysis of Facial Expression: Version 6.0. In: Noldus Information Technology BV Wageningen, the Netherlands.

Pedersen, C., Togelius, J., \& Yannakakis, G. N. (2010). Modeling player experience for content creation. IEEE Transactions on Computational Intelligence and AI in Games, 2(1), 54-67.

Peever, N., Johnson, D., \& Gardner, J. (2012). Personality \& video game genre preferences. Paper presented at the Proceedings of the 8th australasian conference on interactive entertainment: Playing the system.

Perron, B. (2005). A cognitive psychological approach to gameplay emotions.

Pervin, L. A., Cervone, D., \& Oliver, P. (2005). Theories of personality. Personality: Theory and research.

Petrides, K. V. (2009). Psychometric properties of the trait emotional intelligence questionnaire (TEIQue). In Assessing emotional intelligence (pp. 85-101): Springer.

Petrovica, S. (2017, 3-5 July, 2017\}). MULTI-LEVEL ADAPTATION OF AN EDUCATIONAL GAME TO INDIVIDUAL STUDENT'S GAMEPLAY, KNOWLEDGE AND EMOTIONS. Paper presented at the 9th International Conference on Education and New Learning Technologies, Barcelona, Spain.

Power, M., \& Dalgleish, T. (1997). Cognition and Emotion From Order to Disorder. (Hove: Psychology Press. ed. Vol. ).

Psaltis, A., Kaza, K., Stefanidis, K., Thermos, S., Apostolakis, K. C., Dimitropoulos, K., \& Daras, P. (2016). Multimodal affective state recognition in serious games applications. Paper presented at the Imaging Systems and Techniques (IST), 2016 IEEE International Conference on.

Rasim, Langi, A. Z., Munir, \& Rosmansyah, Y. (2016). A survey on adaptive engine technology for serious games. Paper presented at the AIP Conference Proceedings.

Ravaja, N., Saari, T., Laarni, J., Kallinen, K., Salminen, M., Holopainen, J., \& Järvinen, A. (2005). 1 The Psychophysiology of Video Gaming: Phasic Emotional Responses to Game Events.

Ravaja, N., Saari, T., Salminen, M., Laarni, J., \& Kallinen, K. (2006). Phasic emotional reactions to video game events: A psychophysiological investigation. Media Psychology, 8(4), 343-367.

Ravaja, N., Turpeinen, M., Saari, T., Puttonen, S., \& Keltikangas-Järvinen, L. (2008). The psychophysiology of James Bond: Phasic emotional responses to violent video game events. Emotion, 8(1), 114.

Schrader, C., Brich, J., Frommel, J., Riemer, V., \& Rogers, K. (2017). Rising to the challenge: An emotiondriven approach toward adaptive serious games. In Serious Games and Edutainment Applications (pp. 3-28): Springer.

Schrader, C., \& Nett, U. (2017). The perception of control as a predictor of emotional trends during gameplay. Learning and Instruction.

Shaker, N., Asteriadis, S., Yannakakis, G. N., \& Karpouzis, K. (2011). A game-based corpus for analysing the interplay between game context and player experience. In Affective Computing and Intelligent Interaction (pp. 547-556): Springer.

Shiota, M. N., Keltner, D., \& John, O. P. (2006). Positive emotion dispositions differentially associated with Big Five personality and attachment style. The Journal of Positive Psychology, 1(2), 61-71.

Shute, V. J. (2011). Stealth assessment in computer-based games to support learning. Computer games and instruction, 55(2), 503-524. 
Siegling, A. B., Vesely, A. K., Petrides, K., \& Saklofske, D. H. (2015). Incremental validity of the trait emotional intelligence Questionnaire-Short form (TEIQue-SF). Journal of personality assessment, 97(5), 525-535.

Steunebrink, B. R., Dastani, M., \& Meyer, J.-J. C. (2009). A formal model of emotion-based action tendency for intelligent agents. Paper presented at the Portuguese Conference on Artificial Intelligence.

Szilas, N., \& Acosta, M. (2011). A theoretical background for educational video games: Games, signs, knowledge. Handbook of Research on Improving Learning and Motivation through Educational Games: Multidisciplinary Approaches, Hershey, PA: IGI Global, 215-238.

Tallim, J. (2004). What video games have to teach us about learning and literacy. In: JSTOR.

Tijs, T., Brokken, D., \& IJsselsteijn, W. (2008). Creating an emotionally adaptive game. Paper presented at the International Conference on Entertainment Computing.

Tognetti, S., Garbarino, M., Bonarini, A., \& Matteucci, M. (2010). Modeling enjoyment preference from physiological responses in a car racing game. Paper presented at the Computational Intelligence and Games (CIG), 2010 IEEE Symposium on.

Wang, H., \& Sun, C.-T. (2011). Game reward systems: Gaming experiences and social meanings. Paper presented at the DiGRA Conference.

Wiklund, M., Rudenmalm, W., Norberg, L., Westin, T., \& Mozelius, P. (2015). Evaluating educational games using facial expression recognition software: measurement of gaming emotion. Paper presented at the European Conference on Games Based Learning.

Yannakakis, G. N., Isbister, K., Paiva, A., \& Karpouzis, K. (2014). Guest Editorial: Emotion in Games. IEEE Trans. Affective Computing, 5(1), 1-2.

Yannakakis, G. N., \& Togelius, J. (2011). Experience-driven procedural content generation. IEEE Transactions on Affective Computing, 2(3), 147-161.

Yannakakis, G. N., Togelius, J., Khaled, R., Jhala, A., Karpouzis, K., Paiva, A., \& Vasalou, A. (2010). Siren: Towards Adaptive Serious Games for Teaching Conflict Resolution. Paper presented at the 4th European Conference on Games Based Learning, ECGBL 2010.

Yee, N. (2006). Motivations for play in online games. CyberPsychology \& behavior, 9(6), 772-775. 
Title of the article: A generic and efficient emotion-driven approach toward personalized assessment and adaptation in serious games.

\section{Highlights:}

- We proposed an emotion recognition (ER) method/algorithm to assess the player emotion.

- We proposed a method and its algorithm for emotion-driven adaptation (EA).

- Personality and playing style of the player are proposed to personalize both ER and EA.

- We proposed a general architecture of affective serious games.

- To evaluate proposals, a practical experiment is showed. 